\title{
Extended Boussinesq Equations for Rapidly Varying Topography
}

\author{
Gunwoo Kimª, Changhoon Lee ${ }^{\mathrm{b} *}$, Kyung-Duck $\operatorname{Suh}^{\mathrm{c}}$
}

a Coastal Engineering \& Ocean Energy Research Department, Korea Ocean Research

\& Development Institute, Ansan, Gyunggi 426-744, Republic of Korea

b Department of Civil and Environmental Engineering, Sejong University, 98 Kunjadong, Kwangjin-gu, Seoul 143-747, Republic of Korea

c Department of Civil and Environmental Engineering \& Engineering Research

Institute, Seoul National University, 599 Gwanangno, Gwanak-gu, Seoul 151-744, Republic of Korea

* Corresponding author. Fax: +82-2-3408-4332.

E-mail addresses: gwkim@kordi.re.kr (G. Kim), clee@sejong.ac.kr (C. Lee), kdsuh@snu.ac.kr (K.-D. Suh). 


\begin{abstract}
We developed a new Boussinesq-type model which extends the equations of Madsen and Sørensen (1992) by including both bottom curvature and squared bottom slope terms. Numerical experiments were conducted for wave reflection from the Booij's (1983) planar slope with different wave frequencies using several types of Boussinesq equations and extended mild-slope equation. Madsen and Sørensen's model results are accurate in the whole slopes in shallow waters but inaccurate in intermediate water depths. Nwogu's (1993) model results are accurate up to $1: 1(\mathrm{~V}: \mathrm{H})$ slope but significantly inaccurate for steep slopes. The present model results are accurate up to the slope of 1:1 but somewhat inaccurate for very steep slopes. Further, numerical experiments were conducted for wave reflections from a ripple patch and also a Gaussian shaped trench. For the two cases, the results of Nwogu's model and the present model are accurate because these models include the bottom curvature term which is important for the cases. However, Madsen and Sørensen's model results are inaccurate because this model neglects the bottom curvature term.
\end{abstract}

Keywords: Extended Boussinesq equations, higher-order bottom variation terms, numerical experiment, planar slope test, ripple test, Gaussian shaped trench test.

\title{
1. Introduction
}

The transformation of linear regular waves may be predicted using the mild-slope equation of Berkhoff (1972). In deriving the equation, he assumed bottom variation to be small, i.e. $|\nabla h| / k h<<1 \quad(\nabla$ is the horizontal gradient operator, $h$ is the still water depth, $k$ is the wave number), and neglected higher-order bottom variation terms proportional to the squared bottom 
slope, $|\nabla h|^{2}$, and to the bottom curvature, $\nabla^{2} h$. Since he developed an elliptic equation, several researchers have developed different types of mild-slope equations, i.e., parabolic (Radder, 1979; Kirby, 1986) and hyperbolic equations (Nishimura et al., 1983; Copeland, 1985). Also, hyperbolic equations have been developed for irregular waves of narrow frequency band (Smith and Sprinks, 1975; Radder and Dingemans, 1985; Kubo et al., 1992). Since 1990s, by including the higher-order bottom variation terms in the mild-slope equation, several researchers developed modified or extended mild-sloped equations in either elliptic (Massel, 1993; Chamberlain and Porter, 1995) or hyperbolic type (Suh et al., 1997; Lee et al., 1998; Lee et al., 2003b). These models have been found to yield more accurate solutions than the mild-slope equations, in particular, for a rapidly varying topography such as a steep slope or a ripple patch.

Another type of wave model is the Boussinesq equations which may predict the transformation of nonlinear random waves in shallow water. In the Boussiensq equations, the normalization of variables by a typical water depth $\left(h_{0}\right)$ and wave number $\left(k_{0}\right)$ in vertical and horizontal scales, respectively, leads to a mild-slope assumption, i.e. $O(\nabla h)=O\left(k_{0} h_{0}\right)<<1$ (Peregrine, 1972). Thus, Peregrine's (1967) conventional Boussinesq equations which were developed by truncating terms of $O\left(k_{0} h_{0}\right)^{4}$ neglect the squared bottom slope terms but they include the bottom curvature terms. In 1990s the so-called extended Boussinesq equations have been developed to extend the use of Bousssinesq equations to deeper waters. In improving the dispersion relation in deeper waters, Madsen and Sørensen (1992) added spatial derivative terms of the linear shallow water equation with a correction factor to the Peregrine's dispersive terms. By assuming the bottom variation is small, they neglected all the terms of bottom curvature and squared bottom slope. Nwogu (1993) improved the dispersion relation using horizontal velocities at a certain level instead of depth-averaged velocities and truncated the terms of $O\left(k_{0} h_{0}\right)^{4}$ in the equations. Thus, in Nwogu's model the squared bottom slope term is neglected whereas the bottom curvature term 
is included. Gobbi et al. (2000) followed Nwogu's method to improve the dispersion relation and kept the terms up to $O\left(k_{0} h_{0}\right)^{4}$. Even though the squared bottom slope terms as well as the bottom curvature terms are included, Gobbi et al.'s model has so many terms with up to $6^{\text {th }}$-order spatial derivative that it has not been used widely. It was found that in deep waters the extended Boussinesq equations are not accurate in view of both the linear dispersion and shoaling properties (Lee et al., 2003). Further, the extended Boussinesq equations have incorporated linear characteristics far better than nonlinear characteristics. Agnon et al. (1999) achieved the same accuracy in nonlinear properties as well as linear properties. They combined an exact formulation of the boundary conditions at the free surface and at the sea bottom with an approximate solution to the Laplace equation given in terms of truncated series expansions from the still water level. However, the developed model does not provide an accurate vertical distribution of the velocity field. Madsen et al. (2003) solved this problem by following Agnon et al.'s approach but with series expansions from a certain level $z=\hat{z}(x, y)$. They assumed a mildly sloping bottom and included up to the first derivative of $\hat{z}$. Later, Madsen et al. (2006) developed a new Boussinesq equations for rapidly varying bathymetry by including terms proportional to $\nabla \hat{z}, \nabla^{2} \hat{z}$, and $|\nabla \hat{z}|^{2}$. They showed that the developed model are accurate up to the slope of $1: 1(\mathrm{~V}: \mathrm{H})$ on the Booij's (1983) sloping plane.

In this study, we develop Boussiensq equations for a rapidly varying topography by following Madsen and Sørensen's (1992) approach while including both the bottom curvature and squared bottom slope terms which were neglected by them. The developed model's accuracy is verified by comparing the reflection coefficients of waves over the Booij's (1983) planar slope, the Davies and Heathershaw's (1984) ripple, and the Bender and Dean's (2003) Gaussian shaped trench against the exact solutions or experimental data. 


\section{Development of Extended Boussinesq Equations}

The conventional Boussinesq equations of Peregrine (1967) were derived based on the assumption of an incompressible and inviscid fluid. The variables are normalized as

$$
\begin{aligned}
& \left(x^{\prime}, y^{\prime}\right)=\left(k_{0} x, k_{0} y\right), \quad z^{\prime}=z / h_{0}, \quad t^{\prime}=\omega_{0} t=\sqrt{g h_{0}} k_{0} t, \quad h^{\prime}=h / h_{0} \\
& \eta^{\prime}=\eta / a_{0}, \quad \phi^{\prime}=\left(\frac{k_{0} h_{0}}{a_{0} \sqrt{g h_{0}}}\right) \phi
\end{aligned}
$$

where the prime implies that the variable is normalized, $h_{0}$ is the still water depth, $a_{0}$ is the wave amplitude, and $k_{0}$ is the wave number. A normalized Laplace's equation was given by

$$
\mu^{2} \nabla^{\prime 2} \phi^{\prime}+\frac{\partial^{2} \phi^{\prime}}{\partial z^{\prime 2}}=0, \quad-h^{\prime}<z^{\prime}<\delta \eta^{\prime}
$$

And, normalized boundary conditions at the bottom and the water surface were given by

$$
\begin{array}{ll}
\mu^{2} \nabla^{\prime} h^{\prime} \cdot \nabla^{\prime} \phi^{\prime}+\frac{\partial \phi^{\prime}}{\partial z^{\prime}}=0, & z^{\prime}=-h^{\prime} \\
\mu^{2}\left(\eta^{\prime}+\frac{\partial \phi^{\prime}}{\partial t^{\prime}}\right)+\frac{\delta}{2}\left[\mu^{2}\left|\nabla^{\prime} \phi^{\prime}\right|^{2}+\left(\frac{\partial \phi^{\prime}}{\partial z^{\prime}}\right)^{2}\right]=0, & z^{\prime}=\delta \eta^{\prime} \\
\mu^{2}\left(\frac{\partial \eta^{\prime}}{\partial t^{\prime}}+\delta \nabla^{\prime} \eta^{\prime} \cdot \nabla^{\prime} \phi^{\prime}\right)-\frac{\partial \phi^{\prime}}{\partial z^{\prime}}=0, & z^{\prime}=\delta \eta^{\prime}
\end{array}
$$

where $\mu=k_{0} h_{0}$ and $\delta=a_{0} / h_{0}$ denote the frequency dispersion and nonlinearity parameters, respectively. Peregrine developed the Boussinesq equations based on the assumption of a shallow water and a weakly nonlinear wave (i.e., $O(\mu)<<1$ and $O(\delta)<<1$, respectively) and the 
relation of $O(\delta)=O\left(\mu^{2}\right)$. Thus, the bottom slope has the order of magnitude as

$$
\nabla h=\mu \nabla^{\prime} h^{\prime}=O(\mu)
$$

which implies that the bottom slope is mild. Peregrine's equations can be written in terms of the water surface elevation and depth-integrated velocities as

$$
\begin{aligned}
& \frac{\partial \eta}{\partial t}+\frac{\partial P}{\partial x}+\frac{\partial Q}{\partial y}=0 \\
& \frac{\partial P}{\partial t}+\frac{\partial}{\partial x}\left(\frac{P^{2}}{d}\right)+\frac{\partial}{\partial y}\left(\frac{P Q}{d}\right)+g d \frac{\partial \eta}{\partial x}+\psi_{x P}=0 \\
& \frac{\partial Q}{\partial t}+\frac{\partial}{\partial y}\left(\frac{Q^{2}}{d}\right)+\frac{\partial}{\partial x}\left(\frac{P Q}{d}\right)+g d \frac{\partial \eta}{\partial y}+\psi_{y P}=0
\end{aligned}
$$

where $\eta$ is the surface elevation, $d(=h+\eta)$ is the total water depth, $h$ is the still water depth, $P$ and $Q$ are the depth-integrated velocity components in the $x$ - and $y$-directions, respectively, and $\psi_{x P}$ and $\psi_{y P}$ are dispersive terms expressed by

$$
\begin{aligned}
& \psi_{x P}=\frac{1}{6} h^{3}\left\{\frac{\partial^{3}}{\partial x^{2} \partial t}\left(\frac{P}{h}\right)+\frac{\partial^{3}}{\partial x \partial y \partial t}\left(\frac{Q}{h}\right)\right\}-\frac{1}{2} h^{2}\left\{\frac{\partial^{3} P}{\partial x^{2} \partial t}+\frac{\partial^{3} Q}{\partial x \partial y \partial t}\right\} \\
& \psi_{y P}=\frac{1}{6} h^{3}\left\{\frac{\partial^{3}}{\partial y^{2} \partial t}\left(\frac{Q}{h}\right)+\frac{\partial^{3}}{\partial x \partial y \partial t}\left(\frac{P}{h}\right)\right\}-\frac{1}{2} h^{2}\left\{\frac{\partial^{3} Q}{\partial y^{2} \partial t}+\frac{\partial^{3} P}{\partial x \partial y \partial t}\right\}
\end{aligned}
$$

where the subscript $P$ means the model of Peregrine. In deriving the equations, he kept the terms of $O\left(\delta, \mu^{2}\right)$ and neglected higher-order terms. The squared bottom slope terms are of $O\left(\mu^{4}\right)$ and thus were neglected in the dispersive terms given by Eqs. (10a) and (10b). However, the bottom curvature terms were included in the dispersive terms.

Madsen and Sørensen (1992) derived the extended Boussinesq equations by adding the 
dispersive terms to the Boussinesq equation of Peregrine (1967). They assumed a mild slope, and thus the higher-order bottom variation terms proportional to $|\nabla h|^{2}$ and $\nabla^{2} h$ were neglected. In this study, we derive the extended Boussinesq equations following Madsen and Sørensen’s (1992) approach but including these higher-order bottom effect terms.

The momentum equations in a weakly nonlinear and weakly dispersive water wave (i.e., $O(\delta)<<1$ and $O(\mu)<<1$ ) may be approximately expressed as

$$
\begin{aligned}
& \frac{\partial P}{\partial t}+g h \frac{\partial \eta}{\partial x}=O\left(\delta, \mu^{2}\right) \\
& \frac{\partial Q}{\partial t}+g h \frac{\partial \eta}{\partial y}=O\left(\delta, \mu^{2}\right)
\end{aligned}
$$

Taking spatial derivatives of the equations gives the following equations

$$
\begin{aligned}
& \frac{\partial^{3} P}{\partial x^{2} \partial t}+g \frac{\partial^{2}}{\partial x^{2}}\left(h \frac{\partial \eta}{\partial x}\right)=O\left(\frac{\delta \mu^{2}}{h^{2}}, \frac{\mu^{4}}{h^{2}}\right) \\
& \frac{\partial^{3} P}{\partial x \partial y \partial t}+g \frac{\partial^{2}}{\partial x \partial y}\left(h \frac{\partial \eta}{\partial x}\right)=O\left(\frac{\delta \mu^{2}}{h^{2}}, \frac{\mu^{4}}{h^{2}}\right) \\
& \frac{\partial^{3} Q}{\partial y^{2} \partial t}+g \frac{\partial^{2}}{\partial y^{2}}\left(h \frac{\partial \eta}{\partial y}\right)=O\left(\frac{\delta \mu^{2}}{h^{2}}, \frac{\mu^{4}}{h^{2}}\right) \\
& \frac{\partial^{3} Q}{\partial x \partial y \partial t}+g \frac{\partial^{2}}{\partial x \partial y}\left(h \frac{\partial \eta}{\partial y}\right)=O\left(\frac{\delta \mu^{2}}{h^{2}}, \frac{\mu^{4}}{h^{2}}\right)
\end{aligned}
$$

Multiplying Eqs. (12a-d) by $-B h^{2}$ yields the modified terms of $O\left(\delta \mu^{2}, \mu^{4}\right)$. Adding the modified Eqs. (12a) and (12d) to $\psi_{x P}$, and also adding the modified Eqs. (12b) and (12c) to $\psi_{y P}$ give new dispersive terms of $\psi_{x}$ and $\psi_{y}$ given by 


$$
\begin{aligned}
\psi_{x}= & \frac{1}{6} h^{3}\left\{\frac{\partial^{3}}{\partial x^{2} \partial t}\left(\frac{P}{h}\right)+\frac{\partial^{3}}{\partial x \partial y \partial t}\left(\frac{Q}{h}\right)\right\}-\frac{1}{2} h^{2}\left\{\frac{\partial^{3} P}{\partial x^{2} \partial t}+\frac{\partial^{3} Q}{\partial x \partial y \partial t}\right\} \\
& -B h^{2}\left\{\frac{\partial^{3} P}{\partial x^{2} \partial t}+g \frac{\partial^{2}}{\partial x^{2}}\left(h \frac{\partial \eta}{\partial x}\right)+\frac{\partial^{3} Q}{\partial x \partial y \partial t}+g \frac{\partial^{2}}{\partial x \partial y}\left(h \frac{\partial \eta}{\partial y}\right)\right\} \\
\psi_{y}= & \frac{1}{6} h^{3}\left\{\frac{\partial^{3}}{\partial y^{2} \partial t}\left(\frac{Q}{h}\right)+\frac{\partial^{3}}{\partial x \partial y \partial t}\left(\frac{P}{h}\right)\right\}-\frac{1}{2} h^{2}\left\{\frac{\partial^{3} Q}{\partial y^{2} \partial t}+\frac{\partial^{3} P}{\partial x \partial y \partial t}\right\} \\
& -B h^{2}\left\{\frac{\partial^{3} P}{\partial x \partial y \partial t}+g \frac{\partial^{2}}{\partial x \partial y}\left(h \frac{\partial \eta}{\partial x}\right)+\frac{\partial^{3} Q}{\partial y^{2} \partial t}+g \frac{\partial^{2}}{\partial y^{2}}\left(h \frac{\partial \eta}{\partial y}\right)\right\}
\end{aligned}
$$

Eqs. (7), (8), (9) with the dispersive terms $\psi_{x P}$ and $\psi_{y P}$ replaced by $\psi_{x}$ and $\psi_{y}$ in Eqs. (13a) and (13b), respectively, are the extended Boussinesq equation for a rapidly varying topography. If $\nabla^{2} h=|\nabla h|^{2}=0$, Eqs. (13a-b) reduce to the dispersive terms of Madsen and Sørensen (1992) as

$$
\begin{aligned}
\psi_{x M} & =-\left(B+\frac{1}{3}\right) h^{2}\left(\frac{\partial^{3} P}{\partial x^{2} \partial t}+\frac{\partial^{3} Q}{\partial x \partial y \partial t}\right)-B g h^{3}\left(\frac{\partial^{3} \eta}{\partial x^{3}}+\frac{\partial^{3} \eta}{\partial x \partial y^{2}}\right) \\
& -h \frac{\partial h}{\partial x}\left(\frac{1}{3} \frac{\partial^{2} P}{\partial x \partial t}+\frac{1}{6} \frac{\partial^{2} Q}{\partial y \partial t}+2 B g h \frac{\partial^{2} \eta}{\partial x^{2}}+B g h \frac{\partial^{2} \eta}{\partial y^{2}}\right) \\
& -h \frac{\partial h}{\partial y}\left(\frac{1}{6} \frac{\partial^{2} Q}{\partial x \partial t}+B g h \frac{\partial^{2} \eta}{\partial x \partial y}\right) \\
\psi_{y M} & =-\left(B+\frac{1}{3}\right) h^{2}\left(\frac{\partial^{3} Q}{\partial y^{2} \partial t}+\frac{\partial^{3} P}{\partial x \partial y \partial t}\right)-B g h^{3}\left(\frac{\partial^{3} \eta}{\partial y^{3}}+\frac{\partial^{3} \eta}{\partial x^{2} \partial y}\right) \\
& -h \frac{\partial h}{\partial y}\left(\frac{1}{3} \frac{\partial^{2} Q}{\partial y \partial t}+\frac{1}{6} \frac{\partial^{2} P}{\partial x \partial t}+2 B g h \frac{\partial^{2} \eta}{\partial y^{2}}+B g h \frac{\partial^{2} \eta}{\partial x^{2}}\right) \\
& -h \frac{\partial h}{\partial x}\left(\frac{1}{6} \frac{\partial^{2} P}{\partial y \partial t}+B g h \frac{\partial^{2} \eta}{\partial x \partial y}\right)
\end{aligned}
$$

where the subscript $M$ denotes the model of Madsen and Sørensen.

Madsen et al. (1991) suggested the tuning parameter of $B=1 / 15$ which gives a $(2,2)$ Padé approximation of the dispersion relation of linear Stokes waves. The same parameter can be used 
in the present model because the present equation is identical to the Madsen and Sørensen's model over a constant water depth. The difference between the dispersive terms given by (13a-b) and (14a-b) shows the higher order bottom effects as

$$
\begin{aligned}
\psi_{x}-\psi_{x M}= & -h \frac{\partial^{2} h}{\partial x^{2}}\left(\frac{1}{6} \frac{\partial P}{\partial t}+B g h \frac{\partial \eta}{\partial x}\right)-h \frac{\partial^{2} h}{\partial x \partial y}\left(\frac{1}{6} \frac{\partial Q}{\partial t}+B g h \frac{\partial \eta}{\partial y}\right) \\
& +\frac{1}{3} \frac{\partial P}{\partial t}\left(\frac{\partial h}{\partial x}\right)^{2}+\frac{1}{3} \frac{\partial Q}{\partial t} \frac{\partial h}{\partial x} \frac{\partial h}{\partial y} \\
\psi_{y}-\psi_{y M}= & -h \frac{\partial^{2} h}{\partial y^{2}}\left(\frac{1}{6} \frac{\partial Q}{\partial t}+B g h \frac{\partial \eta}{\partial y}\right)-h \frac{\partial^{2} h}{\partial x \partial y}\left(\frac{1}{6} \frac{\partial P}{\partial t}+B g h \frac{\partial \eta}{\partial x}\right) \\
& +\frac{1}{3} \frac{\partial Q}{\partial t}\left(\frac{\partial h}{\partial y}\right)^{2}+\frac{1}{3} \frac{\partial P}{\partial t} \frac{\partial h}{\partial x} \frac{\partial h}{\partial y}
\end{aligned}
$$

\section{Numerical Experiments}

The developed model includes the higher-order bottom effect terms which are neglected in Madsen and Sørensen's (1992) model. We found that Nwogu's (1993) model includes the bottom curvature term, but neglects the squared bottom slope term. In order to examine the accuracy of several Boussinesq-type models, we conducted numerical experiments for monochromatic waves propagating over a planar slope using different inclinations as done by Booij (1983). Numerical experiments were also conducted to measure the reflection coefficients of waves over the ripple patch of Davies and Heathershaw (1984) and also the Gaussian shaped trench of Bender and Dean (2003).

\subsection{Finite difference method}

Including the source term and the energy dissipation term, the resulting linearized one- 
dimensional equations of Madsen and Sørensen (1992) are given by

$\frac{\partial \eta}{\partial t}+\frac{\partial P}{\partial x}=s_{M}$

$\frac{\partial P}{\partial t}+g h \frac{\partial \eta}{\partial x}-\left(B+\frac{1}{3}\right) h^{2} \frac{\partial^{3} P}{\partial x^{2} \partial t}-B g h^{3} \frac{\partial^{3} \eta}{\partial x^{3}}-\frac{1}{3} h \frac{\partial h}{\partial x} \frac{\partial^{2} P}{\partial x \partial t}$

$-2 B g h^{2} \frac{\partial h}{\partial x} \frac{\partial^{2} \eta}{\partial x^{2}}+\omega D_{s} P=0$

where $\omega$ is the angular frequency. The linearized one-dimensional momentum equation of the developed model is given by

$$
\begin{aligned}
& \frac{\partial P}{\partial t}+g h \frac{\partial \eta}{\partial x}-\left(B+\frac{1}{3}\right) h^{2} \frac{\partial^{3} P}{\partial x^{2} \partial t}-\frac{1}{3} h \frac{\partial h}{\partial x} \frac{\partial^{2} P}{\partial x \partial t}-\frac{h}{6} \frac{\partial^{2} h}{\partial x^{2}} \frac{\partial P}{\partial t} \\
& +\frac{1}{3}\left(\frac{\partial h}{\partial x}\right)^{2} \frac{\partial P}{\partial t}-B g h^{2} \frac{\partial^{2}}{\partial x^{2}}\left(h \frac{\partial \eta}{\partial x}\right)+\omega D_{s} P=0
\end{aligned}
$$

The linearized one-dimensional Nwogu's model equations are given by

$$
\begin{aligned}
& \frac{\partial \eta}{\partial t}+\frac{\partial\left(h u_{\alpha}\right)}{\partial x}+\frac{\partial}{\partial x}\left\{\left(\frac{z_{\alpha}^{2}}{2}-\frac{h^{2}}{6}\right) h \frac{\partial^{2} u_{\alpha}}{\partial x^{2}}+\left(z_{\alpha}+\frac{h}{2}\right) h \frac{\partial^{2}\left(h u_{\alpha}\right)}{\partial x^{2}}\right\}=s_{N} \\
& \frac{\partial u_{\alpha}}{\partial t}+g \frac{\partial \eta}{\partial x}+z_{\alpha}\left\{\frac{z_{\alpha}}{2} \frac{\partial^{3} u_{\alpha}}{\partial^{2} x \partial t}+\frac{\partial^{2}}{\partial x^{2}}\left(h \frac{\partial u_{\alpha}}{\partial t}\right)\right\}+\omega D_{s} u_{\alpha}=0
\end{aligned}
$$

where $z_{\alpha}$ is the elevation in which the horizontal particle velocity $u_{\alpha}$ is defined. In this study we used Nwogu's suggested value of $z_{\alpha}=-0.531 h$ (i.e., $\alpha\left(=\left(z_{\alpha} / h\right)^{2} / 2+z_{\alpha} / h\right)=-0.39$ ) which gives minimal squared relative errors in the phase velocity in the depth range of $0<k_{0} h<\pi$ where $k_{0}$ is the wavenumber in a deep water. In Eqs. (16) and (19), $s_{M}$ and $s_{N}$ are line source terms which are determined in the finite difference scheme as (Lee et al., 2001; 
Kim et al., 2007)

$s=2 \frac{C_{e} \Delta t}{\Delta x} \eta^{I}$

where $\eta^{I}$ is the surface elevations of the target wave. The energy velocity $C_{e}$ is the group velocity for the model equations and is obtained by (Lee and Suh, 1998)

$$
\begin{aligned}
C_{e} & =\frac{\omega}{k}\left[1-\frac{(k h)^{2}}{3\left\{1+B(k h)^{2}\left\{\left\{1+\left(B+\frac{1}{3}\right)(k h)^{2}\right\}\right.\right.}\right], \quad \text { for } s_{M} \\
& =\frac{\omega}{k}\left[1-\frac{(k h)^{2}}{3\left\{1-\alpha(k h)^{2}\left\{\left\{1-\left(\alpha+\frac{1}{3}\right)(k h)^{2}\right\}\right.\right.}\right], \quad \text { for } s_{N}
\end{aligned}
$$

And, the energy dissipation term $D_{s}$ is defined by

$$
D_{s}= \begin{cases}\frac{\exp (d / W)-1}{\exp (1)-1}, & \text { inside the sponge layer } \\ 0, & \text { outside the sponge layer }\end{cases}
$$

where $d$ is the distance from the starting point of the sponge layer and $W=2.5 L$ ( $L$ is wavelength) is the thickness of the sponge layer.

All the Boussinesq-type models are discretized in time by the fourth-order Adams-BashforthMoulton predictor-corrector scheme (Wei and Kirby, 1995). The terms with the $1^{\text {st }}$ order spatial derivative are discretized to $O\left(\Delta x^{4}\right)$. A nine-point filtering technique (Shapiro, 1970) is used to smooth numerical noises in the solution of the Boussinesq equations. 


\subsection{Wave reflection from a planar slope}

We conducted numerical experiments for monochromatic waves propagating over a planar slope each end of which was connected to a constant-depth region. This experiment was first made by Booij (1983) who investigated the accuracy of the mild-slope equation of Berkhoff (1972) by comparing the model's reflection coefficients against the exact solution. Since Booij's tests, many researchers have conducted this experiment to demonstrate their model's accuracy. The computational domain for the numerical test is shown in Fig. 1. The water depths on the upand down-wave sides of the slope are $h_{1}=0.6 \mathrm{~m}$ and $h_{2}=0.2 \mathrm{~m}$, respectively, and the width of the sloping bottom, $b$, is varied so that the bottom slope varies. Tests were conducted with the wave frequency of $f=0.5 \mathrm{~Hz}$, and thus the water depths are relatively intermediate with $k h=0.15 \pi \sim 0.28 \pi$.

Booij showed the result of the mild-slope equation in the slope range of 1:15 4:1(V:H) and compared it against the FEM solution of the Laplace equation which is shown in the slope range of 1:3 4:1. The solution of the Laplace equation can be regarded as an exact solution. Booij argued that the mild-slope equation is accurate up to the slope of 1:3. Suh et al. (1997) compared the time-independent solutions of the mild-slope equation (Berkhoff, 1972) and their extended mild-slope equation against the FEM solutions in the same slope range of 1:15 4:1. They found that the extended mild-slope equation is accurate in the whole slope range while the mild-slope equation is not accurate even for mild slopes. They also found that, with the inclusion of the bottom curvature term, the mild-slope equation is accurate up to the slope of 1:1. Lee et al. (1998) also compared the time-dependent solutions of the mild-slope equation (Copeland, 1985) and their extended mild-slope equation against the FEM solutions in the slope range of 1:25 4:1. They obtained almost the same comparisons as Suh et al. (1997). They argued that comparisons of the model equations in view of the bottom slope cannot be made in this condition because of 
the following two reasons. Firstly, this condition did not cover the entire range of water depth from deep to shallow water. Secondly, the solutions were affected by the existence of two slope discontinuities as well as the bottom slope. The slope discontinuities cannot be considered in the mild-slope equations while the extended mild-slope equation considers them with the bottom curvature term.

In this study, we conducted numerical experiments for the Booij's planar slope with different wave frequencies of $f=0.2 \mathrm{~Hz}, 0.35 \mathrm{~Hz}, 0.5 \mathrm{~Hz}, 0.75 \mathrm{~Hz}$, and $1 \mathrm{~Hz}$ to investigate wave reflections in a broad range of relative water depths. Table 1 shows the incident wave frequency and the corresponding relative water depths in the up- and down-wave sides of the slope. For the lowest wave frequency (i.e., $f=0.2 \mathrm{~Hz}$ ), the water depths were shallow with $k h=0.06 \pi \sim 0.10 \pi$ while, for the highest wave frequency (i.e., $f=1 \mathrm{~Hz}$ ), the water depths were intermediate with $k h=0.33 \pi \sim 0.78 \pi$. The reflection coefficient is obtained by

$$
K_{R}=\frac{|\eta|_{\max }-|\eta|_{\min }}{|\eta|_{\max }+|\eta|_{\min }}
$$

where $|\eta|_{\max }$ and $|\eta|_{\min }$ are, respectively, the maximum and minimum values of the wave amplitude $|\eta|$ in a wave envelope which is located between the wave generation point and the starting point of the slope.

Fig. 2 compares FEM solutions of the reflection coefficient at 5 different wave frequencies. As the wave frequency increased, the wave reflections decreased because the bottom was less felt in deeper waters. Also, with the higher wave frequency, there existed more numbers of resonant and non-resonant reflections with the slope-width variation. This could be explained by the Bragg 
$\underline{\text { reflection, which occurs when the relation } 2 k / K=n} \underline{\text { is satisfied, where }} \underline{k}$ and $K \underline{K}$ are the $\underline{\text { wavenumbers of surface wave and bottom undulation, respectively, and }} \underline{n} \underline{\text { is an arbitrary }}$

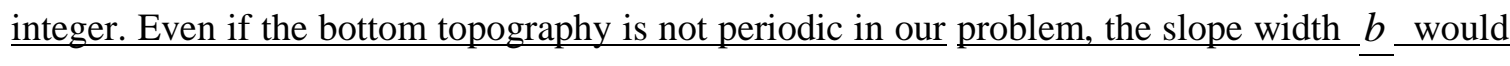
be somehow related to $\underline{K}$. Since $\underline{b}$ varies between 0.1 and $10.0 \mathrm{~m}, \underline{K} \underline{\text { will also vary within a }}$ $\underline{\text { certain range, which does not depend on the wave frequency. However, }} \underline{k \text { increases with the }}$ wave frequency. Therefore, the number of integers which satisfy the relation $2 k / K=n$ increases with the frequency. In other words, the number of the Bragg resonant peaks increases with the frequency, as shown in Fig. 2.

Fig. 3 compares the reflection coefficients calculated by the present equations, Madsen and Sørensen's (1992) equations, and Nwogu's (1993) equations against the FEM solution of the Laplace equation at 5 different wave frequencies. For $f=0.5 \mathrm{~Hz}$, we also compared available solutions of Madsen et al.'s (2006) equations which included higher-order bottom variation effects in the fully nonlinear Boussinsq equations of Madsen et al. (2003). Further, for a quantitative comparison at $f=0.5 \mathrm{~Hz}$, we showed the percent error of the reflection coefficient against the FEM solution in Fig. 4. The percent error is defined as $\left|R_{c}-R_{e}\right| / R_{e} \times 100$ where $R_{c}$ and $R_{e}$ are the calculated reflection coefficient and the FEM solution, respectively. Madsen and Sørensen's equations showed accurate solutions in the whole slope range in shallow waters with $f=0.2 \mathrm{~Hz}$. However, as the water depths became intermediate with $f=0.35 \mathrm{~Hz}, 0.5 \mathrm{~Hz}, 0.75 \mathrm{~Hz}$, and $1 \mathrm{~Hz}$, Madsen and Sørensen's equations overestimated the reflection coefficient even for very mild slopes because they neglected the bottom curvature term as well as the squared bottom slope term (see Fig. 4 for a quantitative comparison at $f=0.5 \mathrm{~Hz}$ ). Nwogu's equations were accurate up to the slope of $1: 1$ in the whole wave frequency range except the highest frequency of $f=1 \mathrm{~Hz}$ because they included the bottom curvature term. However, Nwogu's equations significantly overestimated the reflection coefficient at steeper slopes because they neglected the squared bottom slope term which is important for steeper slopes. The present equations were 
accurate up to the slope of $1: 1$ in the whole wave frequency range because they included the bottom curvature term. However, the present equations overestimated the reflection coefficient at steeper slopes. This inaccuracy can be explained by two reasons. First, the present equations yielded more errors in deeper waters because the parameter of $B=1 / 15$ could not guarantee exact solutions in the linear dispersion and shoaling properties even in intermediate water depths (see detailed accuracies in Figs. 1 and 3 of Lee et al. (2003a)). Second, the present equations did not correctly consider the evanescent modes which became important in intermediate water depths. Fig. 3(e) shows non-negligible errors of the present equation in the whole slope range for the case of $f=1 \mathrm{~Hz}$ in which relative water depths are $0.33 \pi \leq k h \leq 0.78 \pi$. For $f=0.5 \mathrm{~Hz}$, Madsen et al.'s (2006) solution showed a deviation from the exact solution at the slope of 1:1 which was larger than the present solution (see Figs. 3(c) and 4). The trend of inaccuracies implies that for steeper slopes the deviation would be more significant than the present solution.

In order to examine each effect of the squared bottom slope term and the bottom curvature term, additional calculations were made by including each term to Madsen and Sørensen's model at higher wave frequencies of $f=0.5 \mathrm{~Hz}, 0.75 \mathrm{~Hz}$, and $1 \mathrm{~Hz}$. Comparisons were made in Fig. 5 . The inclusion of the squared bottom slope term gave almost identical results to Madsen and Sørensen's model for very mild slopes, and it gave even worse results than Madsen and Sørensen's model for very steep slopes. For very steep slopes, the trend of increase of reflection coefficients with the bottom slope was almost the same as the present equations. It is because the present model also includes the squared bottom slope term which is important for very steep slopes. On the other hand, the inclusion of the bottom curvature term for $f=0.5 \mathrm{~Hz}$ and $0.75 \mathrm{~Hz}$ significantly improved Madsen and Sørensen's model in the whole slope range even more accurate than the present model. This means that the bottom curvature term is more important than the squared bottom slope term in improving the Madsen and Sørensen's model even for 
steeper slopes. However, for $f=1 \mathrm{~Hz}$, the inclusion of the bottom curvature term gave less accurate solutions than the present model. This implies that in a deeper water the equations with both the bottom curvature and squared bottom slope terms gives more accurate solutions than with only the bottom curvature term.

\subsection{Wave reflection from a non-plane slope}

The Booij's(1983) problem discussed in the previous section involves the effect of bottom curvature only at the two points at the ends of the slope. In order to assess the simultaneous influence of the steepness and curvature of slope, Suh et al. (1998) considered a non-plane slope where both the steepness and curvature of the slope vary continuously in space. The water depth on the slope was given by (Fig. 4)

$h(x)=0.5\left(h_{1}+h_{2}\right)-0.5\left(h_{1}-h_{2}\right) \tanh p(x)$ 
Where

$$
p(x)=3 \pi\left(\frac{x}{b}-\frac{1}{2}\right)
$$

$b$ is the width of the slope where the water depth varies. As in the Booij's problem, the constant depths on the upwave and downwave sides of the slope were chosen to be $h_{1}=0.6 \mathrm{~m}$ and $h_{2}=0.2 \mathrm{~m}$, respectively, and the wave period is $2 \mathrm{~s}$.

\subsection{Bragg reflection from a ripple patch}

When surface waves are normally incident on a region of long-crested periodic bottom undulation, a significant amount of incident wave energy is reflected at the point where the wavenumber of the periodic bottom undulation $(K)$ is twice the wavenumber of the surface wave ( $k$ ), that is $2 k / K=1$. This type of wave reflection may be categorized as the Class I Bragg reflection (Liu and Yue, 1998). Davies and Heathershaw (1984) conducted experiments with different numbers of ripples and water depth. In their experiment, the ripple wavelength and amplitude were $1 \mathrm{~m}$ and $5 \mathrm{~cm}$, respectively, and the numbers of ripples were 2,4 , and 10 . The water depth in the constant-depth region was $15.6 \mathrm{~cm}$ for the cases of 2 and 4 ripples and $31.3 \mathrm{~cm}$ for the case of 10 ripples. Their experimental data has been used for comparisons to various numerical models. We also conducted numerical experiments with the condition of Davies and Heathershaw. The computational domain for numerical test is shown in Fig. 6. The water depth is 
given by

$$
h(x)=\left\{\begin{array}{cc}
h_{c} & x \leq x_{r} \\
h_{c}-A \sin \left[K\left(x-x_{r}\right)\right] & x_{r} \leq x \leq x_{r}+n L_{r} \\
h_{c} & x \geq x_{r}+n L_{r}
\end{array}\right.
$$

where $A$ is the ripple amplitude, $L_{r}$ is the ripple wavelength, $n$ is the number of ripples, $h_{c}$ is the water depth at the constant-depth region, $K$ is the wave number of the ripple, and $x_{r}$ is the $x$-coordinate of the starting point of the ripple patch.

Figs. 7(a)-(c) compares the reflection coefficients calculated by the present model, Madsen and Sørensen's model, and Nwogu's model against the experimental data for the cases of 2, 4, and 10 ripples, respectively. For the case of 10 ripples, Madsen et al.'s (2006) model results were also compared. The reflection coefficients calculated by Madsen and Sørensen's model were close to the experimental data for the case of 2 ripples. However, as the number of ripples increased to 4 and 10, the Bragg reflection significantly occurred and Madsen and Sørensen's model yielded reflection coefficient larger than the experimental data. However, Nwogu's model and the present model predicted the Bragg reflection very well for all the three cases. For the case of 10 ripples, Madsen et al.'s model results were on the whole close to the experimental data. However, around the resonance conditions of $2 k / K=1$ and $2 k / K=2$ the predicted reflection coefficients were slightly larger and smaller than the experimental data, respectively. Lee et al. (1998) found that the magnitude of the bottom curvature of the ripple patch (i.e., $\quad L_{r} d^{2} h / d x^{2}=O\left(A / L_{r}\right)$ ) is larger than the magnitude of the squared bottom slope (i.e., $\left.(d h / d x)^{2}=O\left(A / L_{r}\right)^{2}\right)$ due to $A / L_{r}<<1$. This means that for a ripple patch the bottom curvature term is more important than the squared bottom slope term. Madsen and Sørensen's model neglected the bottom curvature term whereas other models included the bottom curvature term. 


\subsection{Wave reflection from a Gaussian shaped trench}

We also conducted numerical experiments for monochromatic waves propagating over a Gaussian shaped trench. This experiment was first made by Bender and Dean (2003) who used the step method to get reflection coefficients over the trench. Later, Madsen et al. (2006) tested their model for the trench and found problematic solutions using a fixed condition of the elevation $\hat{z}=-0.5 h$. Their model has been derived based on the assumption of a mildly varying $\hat{z}$ which is not true for this case. They could get accurate solutions after smoothing the rapidly varying $\hat{z}$. In the test of Booij's (1983) planar slope, Madsen et al. smoothed the elevation $\hat{z}$ at the two ends of the slope when the slope was steeper than 0.2 .

In this study, we tested for the case of a rapidly varying trench in which Madsen et al. smoothed the elevation $\hat{z}$ for accurate solutions. The water depth is given by

$$
h(x)=h_{c}+C_{1} \exp \left[-\frac{\left(x-x_{t}\right)^{2}}{2 C_{2}^{2}}\right]
$$

where $h_{c}$ is the water depth at the constant-depth region, $x_{t}$ is the $x$-coordinate of the starting point of the trench, and $C_{1}$ and $C_{2}$ are the shape parameters. The computational domain for numerical test is shown in Fig. 8. The bottom conditions were given by $h_{c}=10 \mathrm{~m}, C_{1}=7.5 \mathrm{~m}$, $C_{2}=12.5 \mathrm{~m}$

Fig. 9 compares the reflection coefficients calculated by the present equations, Madsen and Sørensen's (1992) equations, and Nwogu's (1993) equations against the Bender and Dean's 
(2003) step method. We also compared Madsen et al.'s (2006) solutions which included the $\delta^{2}$ terms for the rapidly varying topography. Bender and Dean's solution considers both the propagating and evanescent modes and thus can be regarded as the exact solution.

Bender and Dean's solution showed that the reflection coefficient was resonantly peaked at $k_{c} h_{c}=0.13 \pi$ and decreased monotonically in shallower and deeper waters from the water of the resonant reflection. The resonant reflection coefficient calculated by Madsen and Sørensen's model were larger than Bender and Dean's solutions, while the results of other models predicted the resonant reflection very well. The magnitude of the bottom curvature of the trench (i.e., $\left.L_{c} d^{2} h / d x^{2}=O\left(C_{1} / L_{c}\right)\right)$ is larger than the magnitude of the squared bottom slope (i.e., $\left.(d h / d x)^{2}=O\left(C_{1} / L_{c}\right)^{2}\right)$ due to $C_{1} / L_{c}<<1$ where $L_{c}$ is the trench length. The bottom curvature effect was neglected in Madsen and Sørensen's model whereas it was considered in other models.

Near the deeper-water edge of the resonant reflection, the results of Nwogu's model, Madsen and Sørensen's model and the present model overestimated the reflection coefficient. And, the errors were largest in the Nwogu's model and smallest in the present model. These were due to firstly the fact that near the deeper-water edge of $k_{c} h_{c}=0.4 \pi$ (i.e., $0.40 \pi \leq k h \leq 0.62 \pi$ ) Nwogu's model yielded larger errors in the linear dispersion relation and the shoaling properties than Madsen and Sørensen's model and the present model (see Figs. 1 and 3 of Lee et al. (2003a)). Secondly, Madsen and Sørensen's model neglected the bottom curvature effect which was considered in Nwogu's model and the present model.

\section{Conclusions}

In this study, we found that a mild slope assumption was made in most of the Boussinesq 
equations. Thus, both the bottom curvature and squared bottom slope terms were neglected in the equations of Madsen and Sørensen (1992) and the squared bottom slope terms were neglected in the equations of Nwogu (1993). Further, we developed a new model which extends the model of Madsen and Sørensen (1992) by including both the bottom curvature and squared bottom slope terms.

Numerical experiments were conducted to measure reflection coefficients of waves over the Booij's (1983) planar slope using several types of the Boussinesq equations and the extended mild-slope equation. Madsen and Sørensen's model results were accurate in the whole slope range in shallow waters but inaccurate in intermediate water depths. Nwogu's (1993) model results were accurate up to $1: 1(\mathrm{~V}: \mathrm{H})$ slope but significantly inaccurate for steep slopes. The results of the present model were accurate up to the slope of 1:1 but somewhat inaccurate for very steep slopes. Conclusively, the tested Boussinesq equations including one or both of the higherorder bottom effect terms performed well for slopes smaller than unity, which is the maximum slope for most beaches and sloping coastal structures.

Also, numerical experiments were conducted to measure reflection coefficients of waves over the Davies and Heathershaw's (1984) ripple patch and the Bender and Dean's (2003) Gaussian shaped trench. For all the two cases, the solutions of both the Nwogu's model and the present model were accurate because they included the bottom curvature term. However, the solutions of Madsen and Sørensen were inaccurate due to the neglect of the bottom curvature term.

\section{Acknowledgements}

This work was supported by the Korea Science and Engineering Foundation (KOSEF) grant funded by the Korea government (MEST) (No. R01-2008-000-20442-0). 


\section{References}

Agnon, Y., Madsen, P.A., Schäffer, H.A., 1999. A new approach to high-order Boussinesq models. Journal of Fluid Mechanics 399, 319-333.

Bender, C.J., Dean, R.G., 2003. Wave transformation by two-dimensional bathymetric anomalies with sloped transitions. Coastal Engineering 50, 61-84.

Berkhoff, J.C.W., 1972. Computation of combined refraction-diffraction. In: Proceedings of the 13th Coastal Engineering Conference, ASCE, New York, pp. 471-490.

Booij, N., 1983. A note on the accuracy of the mild-slope equation. Coastal Engineering 7, 191203.

Chamberlain, P.G., Porter, D., 1995. The modified mild-slope equation. Journal of Fluid Mechanics 291, 393-407.

Copeland, G.J.M., 1985. A practical alternative to the mild-slope wave equation. Coastal Engineering 9, 125-149.

Davies, A.G., Heathershaw, A.D., 1984. Surface-wave propagation over sinusoidally varying topography. Journal of Fluid Mechanics 144, 419-443.

Gobbi, M.F., Kirby, J.T., Wei, G., 2000. A fully nonlinear Boussinesq model for surface waves. Part 2. Extension to $O(k h)^{4}$. Journal of Fluid Mechanics 405, 181-210.

Kim, G., Lee, C., Suh, K.-D., 2007. Internal generation of waves: delta source function method and source term addition method. Ocean Engineering 34, 2251-2264.

Kirby, J.T., 1986. Rational approximations in the parabolic equation method for water waves. Coastal Engineering 10, 355-378. 
Kubo, Y., Kotake, Y., Isobe, M., Watanabe, A., 1992. Time-dependent mild slope equation for random waves. In: Proceedings of the 23rd International Conference on Coastal Engineering, Venice, ASCE, pp. 419-431.

Lee, C., Cho, Y.-S., Yoon, S.B., 2003a. A note on linear dispersion and shoaling properties in extended Boussinesq equations. Ocean Engineering 30, 1849-1867.

Lee, C., Cho, Y.-S., Yum, K., 2001. Internal generation of waves for extended Boussinesq equations. Coastal Engineering 42, 155-162.

Lee, C., Park, W.S., Cho, Y.-S., Suh, K.D., 1998. Hyperbolic mild-slope equations extended to account for rapidly varying topography. Coastal Engineering 34, 243-257.

Lee, C., Kim, G., Suh, K.D., 2003b. Extended mild-slope equation for random waves. Coastal Engineering 48, 277-287.

Lee, C., Suh, K.D., 1998. Internal generation of waves for time-dependent mild-slope equations. Coastal Engineering 34, 35-57.

Liu, Y., Yue, D.K.P., 1998. On generalized Bragg scattering of surface waves by bottom ripples. Journal of Fluid Mechanics 356, 297- 326.

Madsen, P.A., Bingham, H.B., Schäffer, H.A., 2003. Boussinesq-type formulations for fully nonlinear and extremely dispersive water waves: derivation and analysis. Proceedings of Royal Society of London, A 459, 1075-1104.

Madsen, P.A., Fuhrman, D.R., Wang, B., 2006. A Boussinesq-type method for fully nonlinear waves interacting with a rapidly varying bathymetry. Coastal Engineering 53, 487-504.

Madsen, P.A., Sørensen, O.R., 1992. A new form of the Boussinesq equations with improved linear dispersion characteristics. Part 2. A slowly varying bathymetry. Coastal Engineering 18, 183-204.

Massel, S.R., 1993. Extended refraction-diffraction equation for surface waves, Coastal Engineering 19, 97-126.

Nishimura, H., Maruyama, K., Hirakuchi, H., 1983. Wave field analysis by finite difference 
method. In: Proceedings of the 30th Japanese Conference on Coastal Engineering, pp. 123127 (in Japanese).

Nwogu, O., 1993. Alternative form of Boussinesq equation for nearshore wave propagation. Journal of Waterway, Port, Coastal and Ocean Engineering 119, 618-638.

Peregrine, D.H., 1967. Long waves on a beach. Journal of Fluid Mechanics 27, 815-827.

Peregrine, D.H., 1972. Equations for water waves and the approximation behind them, In: Waves on Beaches and Resulting Sediment Transport, pp. 95-121.

Radder, A.C., 1979. On the parabolic equation method for water-wave propagation. Journal of Fluid Mechanics 95, 159-176.

Radder, A.C., Dingemans, M.W., 1985. Canonical equations for almost periodic, weakly nonlinear gravity waves. Wave Motion 7, 473-485.

Shapiro, R., 1970. Smoothing, filtering, and boundary effects. Review of Geophysics and Space Physics 8(2), 359-386.

Smith, R., Sprinks, T., 1975. Scattering of surface waves by a conical island. Journal of Fluid Mechanics 72, 373-384.

Suh, K.D., Lee, C., Park, W.S., 1997. Time-dependent equations for wave propagation on rapidly varying topography. Coastal Engineering 32, 91-117.

Suh, K.D., Park, W.S., Lee, C., 1998. A note on the modified mild-slope equation. Journal of Korean Society of Coastal and Ocean Engineers 10(2), 55-63.

Wei, G., Kirby, J.T., 1995 Time-dependent numerical code for extended Boussinesq equations, Journal of Waterway, Port, Coastal and Ocean Engineering 121, 251-261. 
Table 1. Incident wave frequency and relative water depths.

\begin{tabular}{|c|c|c|}
\hline frequency (period) & $k_{1} h_{1}$ & $k_{2} h_{2}$ \\
\hline $0.20 \mathrm{~Hz}(5.00 \mathrm{~s})$ & $0.10 \pi$ & $0.06 \pi$ \\
\hline $0.35 \mathrm{~Hz}(2.86 \mathrm{~s})$ & $0.18 \pi$ & $0.10 \pi$ \\
\hline $0.50 \mathrm{~Hz}(2.00 \mathrm{~s})$ & $0.28 \pi$ & $0.15 \pi$ \\
\hline $0.75 \mathrm{~Hz}(1.33 \mathrm{~s})$ & $0.48 \pi$ & $0.23 \pi$ \\
\hline $1.00 \mathrm{~Hz}(1.00 \mathrm{~s})$ & $0.78 \pi$ & $0.33 \pi$ \\
\hline
\end{tabular}




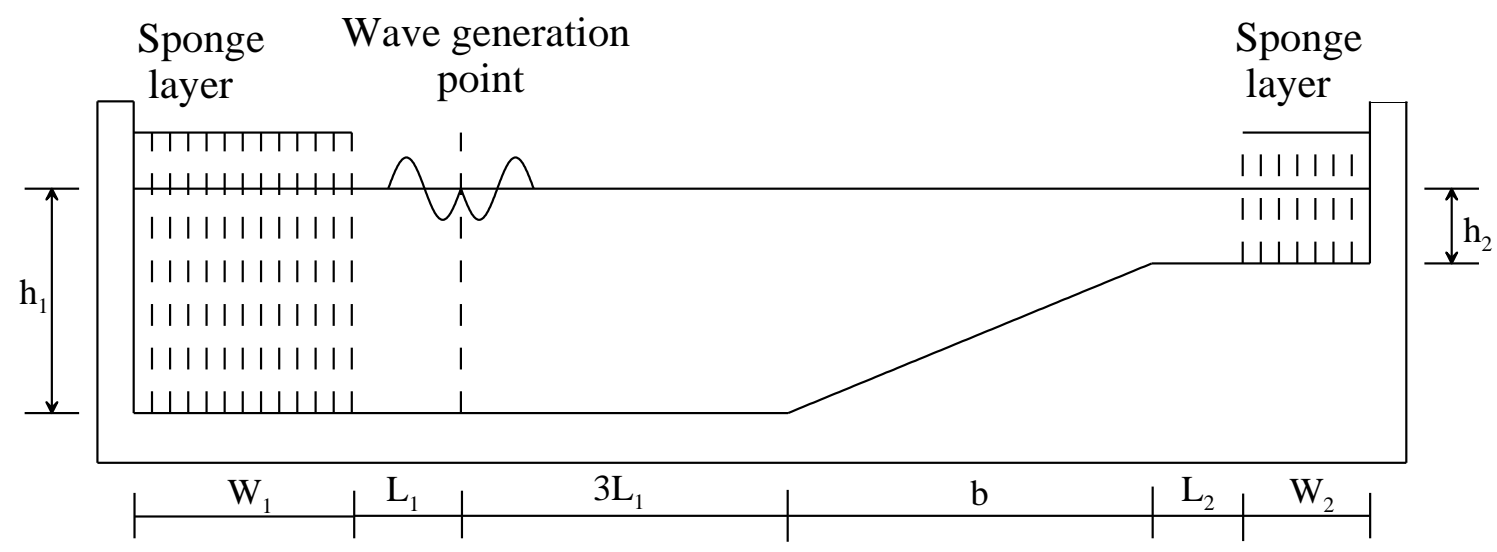

Fig. 1. Computational domain for numerical test of waves propagating over a planar slope. 


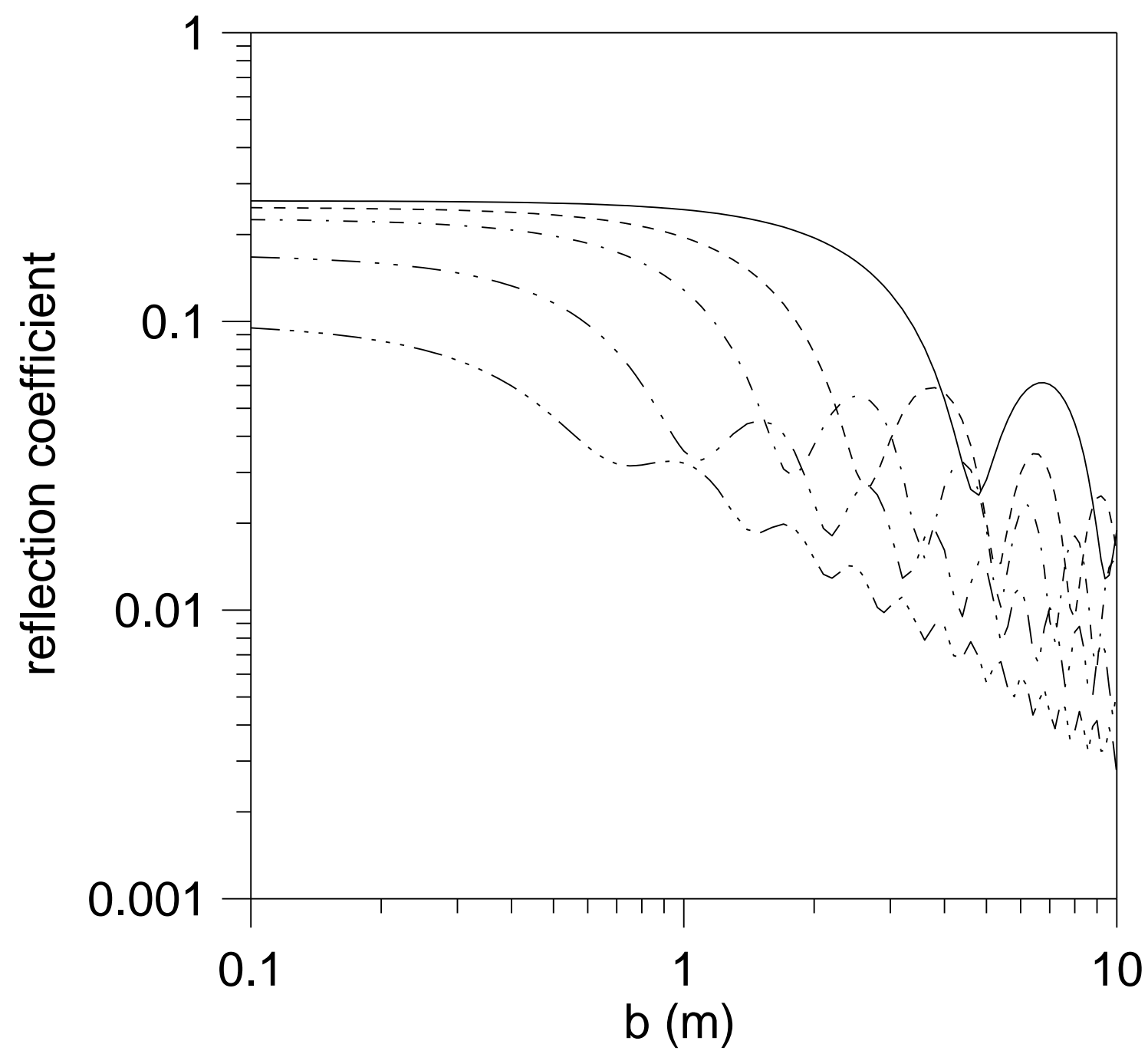

Fig. 2. Variation of FEM solutions of reflection coefficient with width of a planar slope; $-=$ $(\mathrm{f}=0.2 \mathrm{~Hz}),-\cdots-=(\mathrm{f}=0.35 \mathrm{~Hz}), \cdots-\cdots=(\mathrm{f}=0.5 \mathrm{~Hz}),-\cdots-\cdots-=(\mathrm{f}=0.75 \mathrm{~Hz}), \cdots-\cdots-=(\mathrm{f}=1 \mathrm{~Hz})$. 
(a)

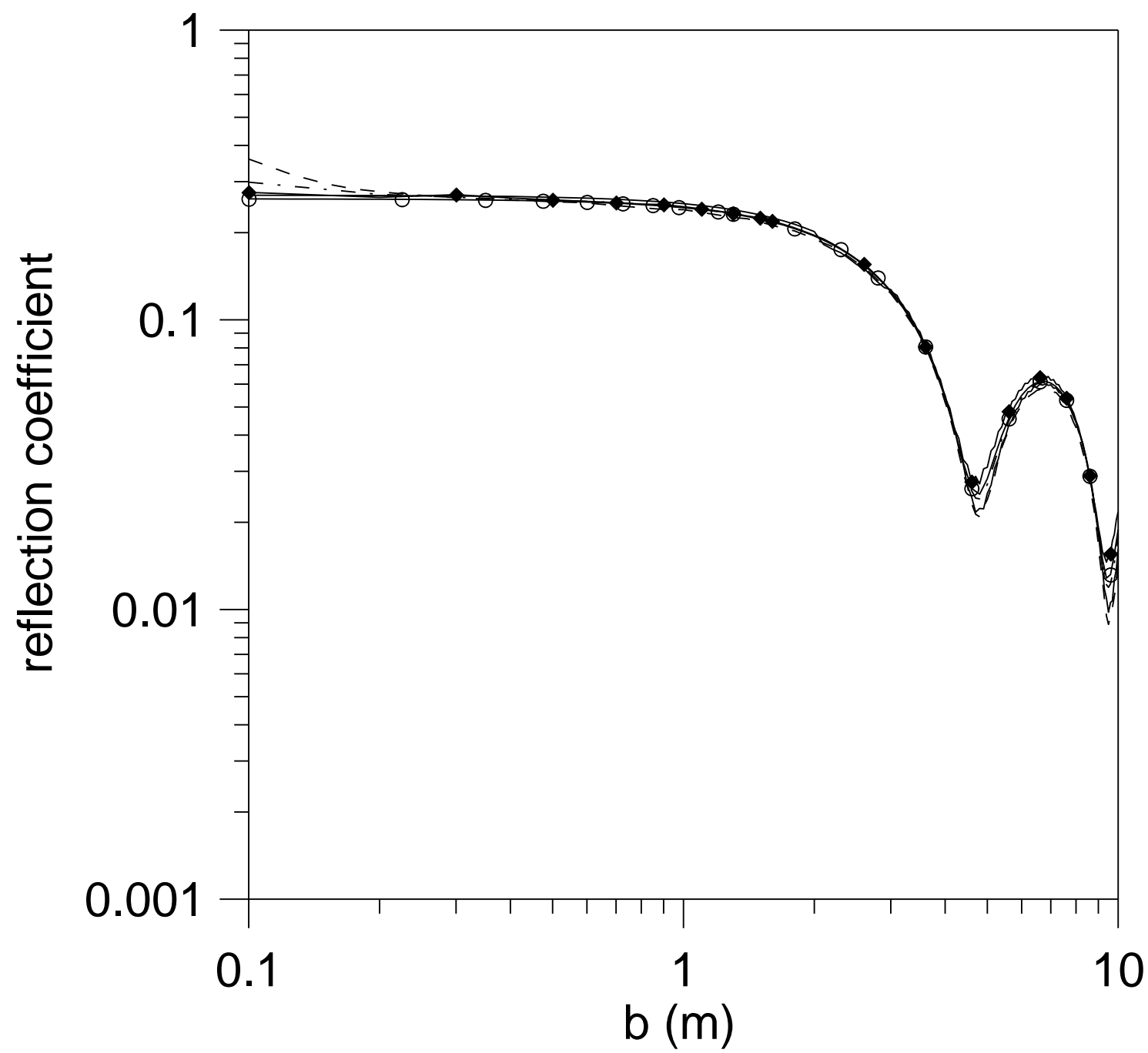

Fig. 3. Variation of reflection coefficients with width of a planar slope: (a) $f=0.2 \mathrm{~Hz}$, (b) $f=0.35$ $\mathrm{Hz}$, (c) $\mathrm{f}=0.5 \mathrm{~Hz}$, (d) $\mathrm{f}=0.75 \mathrm{~Hz}$, (e) $\mathrm{f}=1.0 \mathrm{~Hz}$; —- - = Laplace equation, - - = present equations, $\longrightarrow$ = Madsen and Sørensen (1992), - - - - = Nwogu (1993), — —— = Madsen et al. (2006) (only in Fig. 3(c)). 
(b)

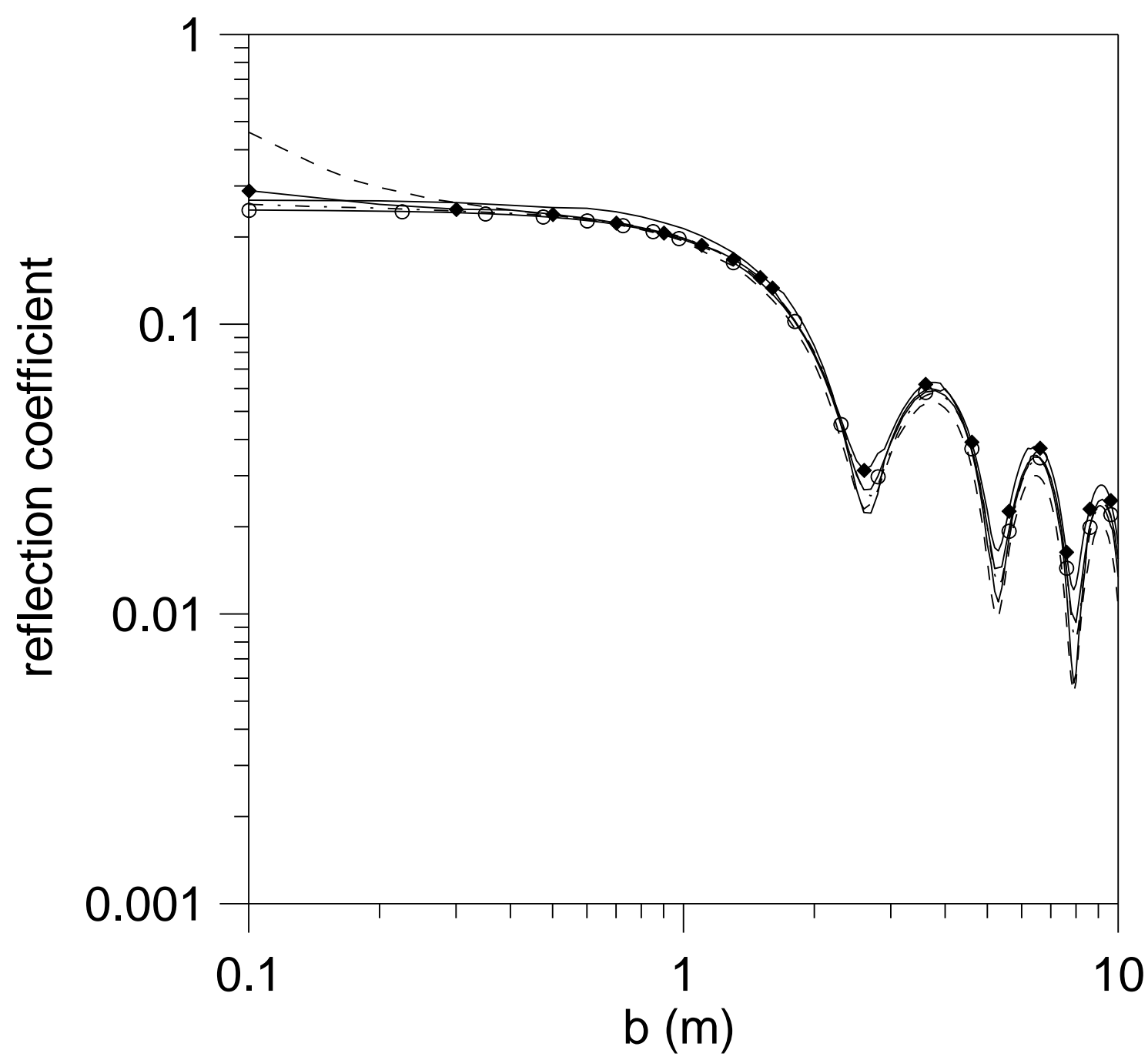

Fig. 3 (continued). 
(c)

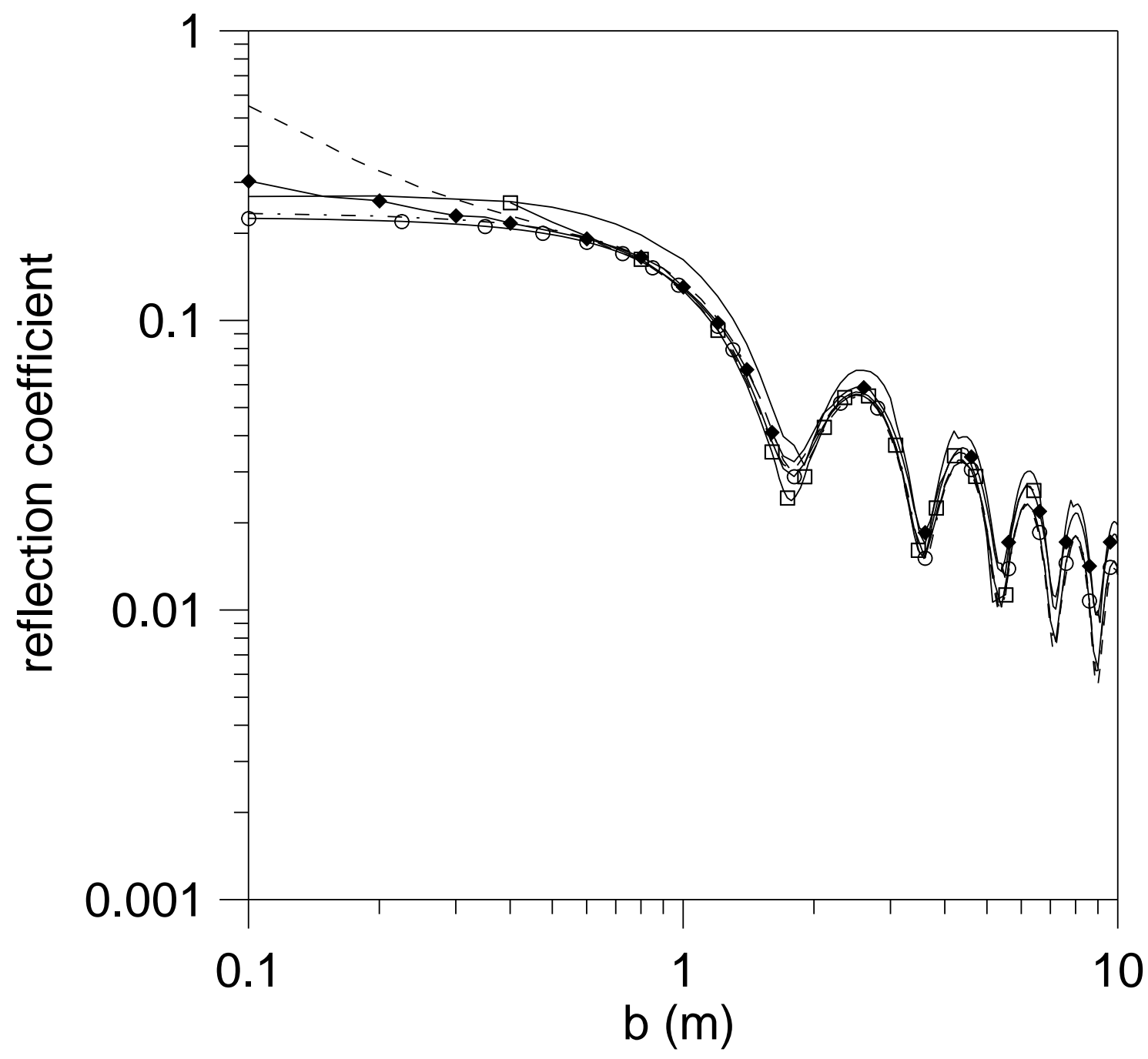

Fig. 3 (continued). 
(d)

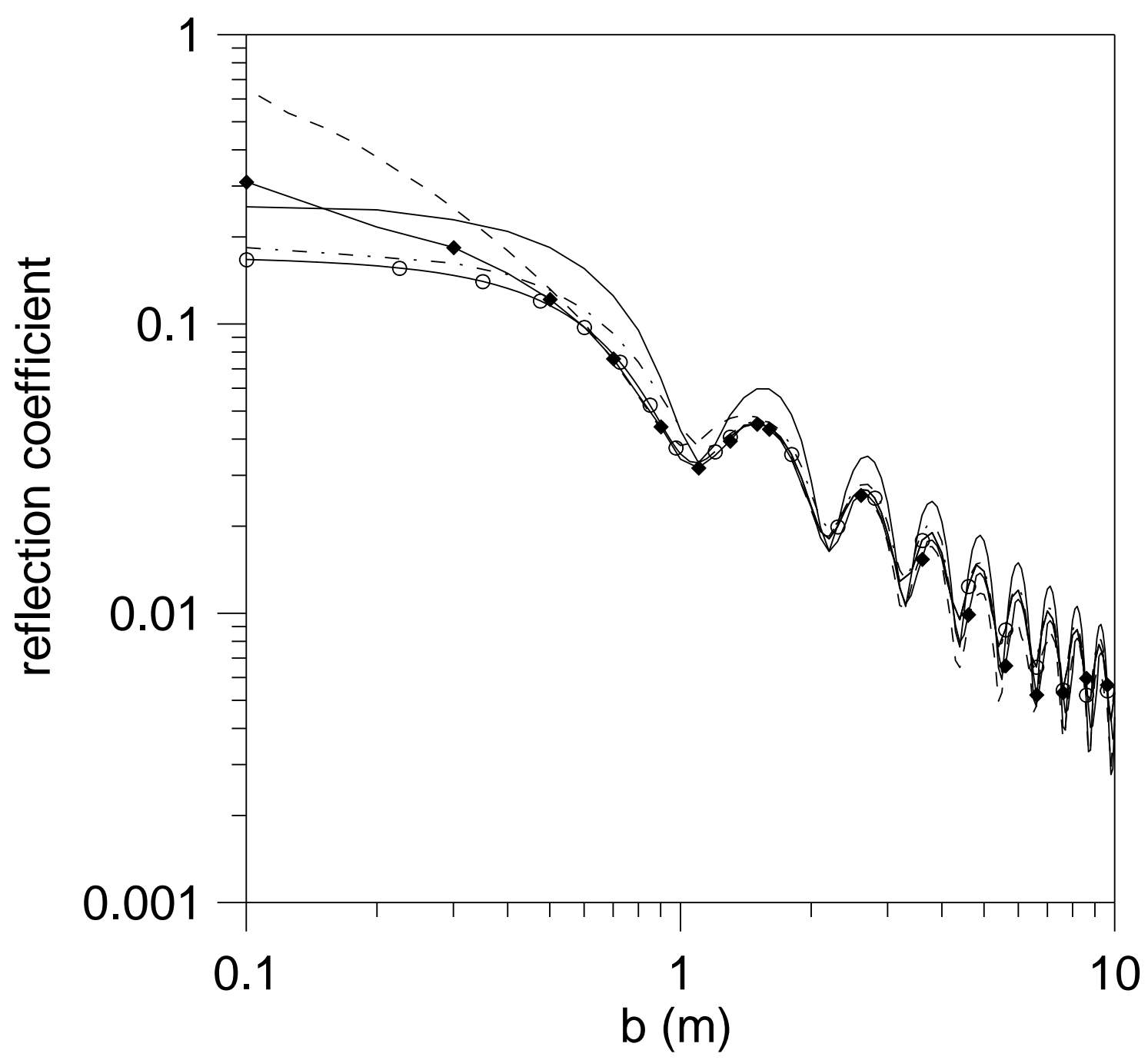

Fig. 3 (continued). 


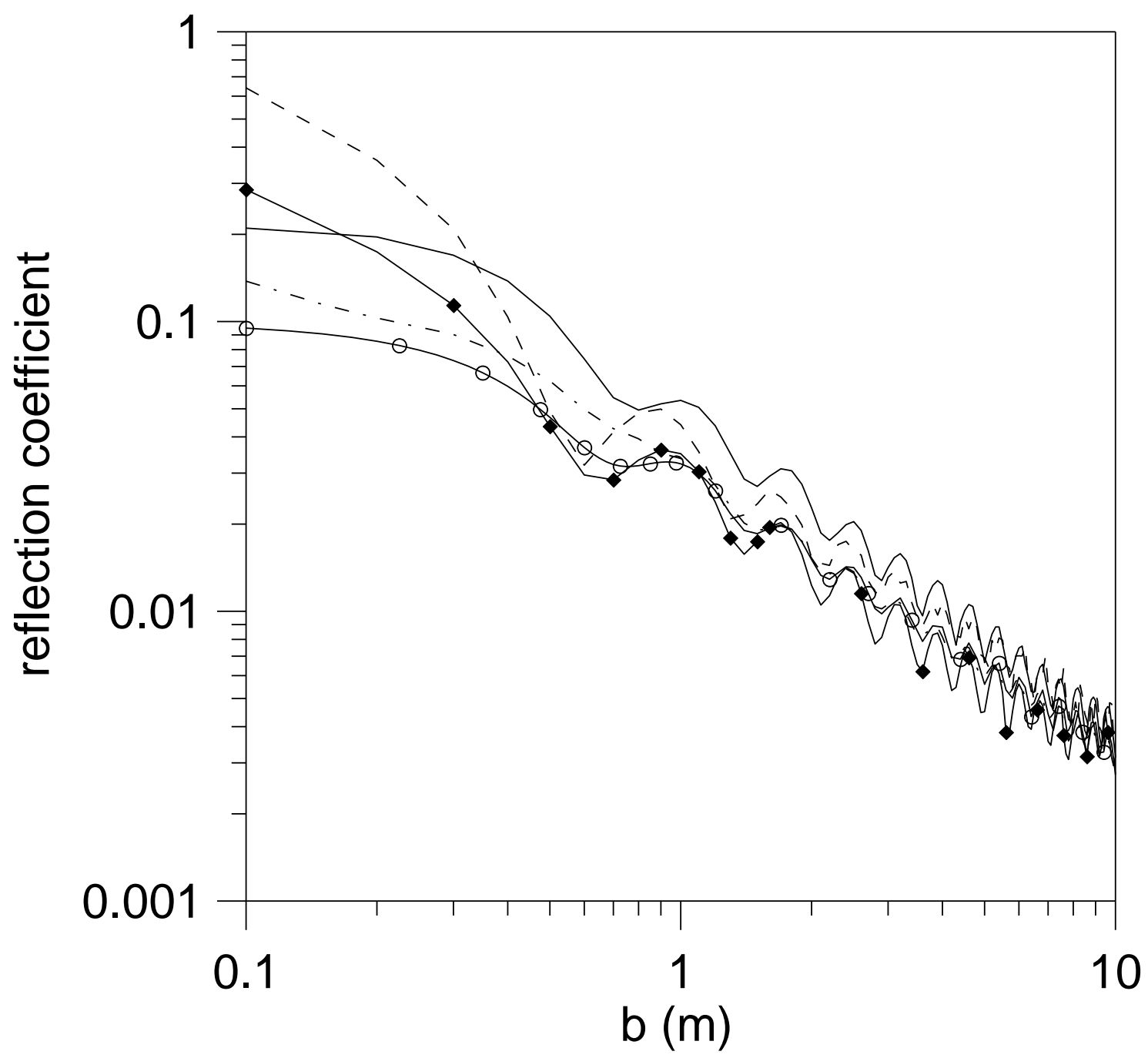

Fig. 3 (continued). 


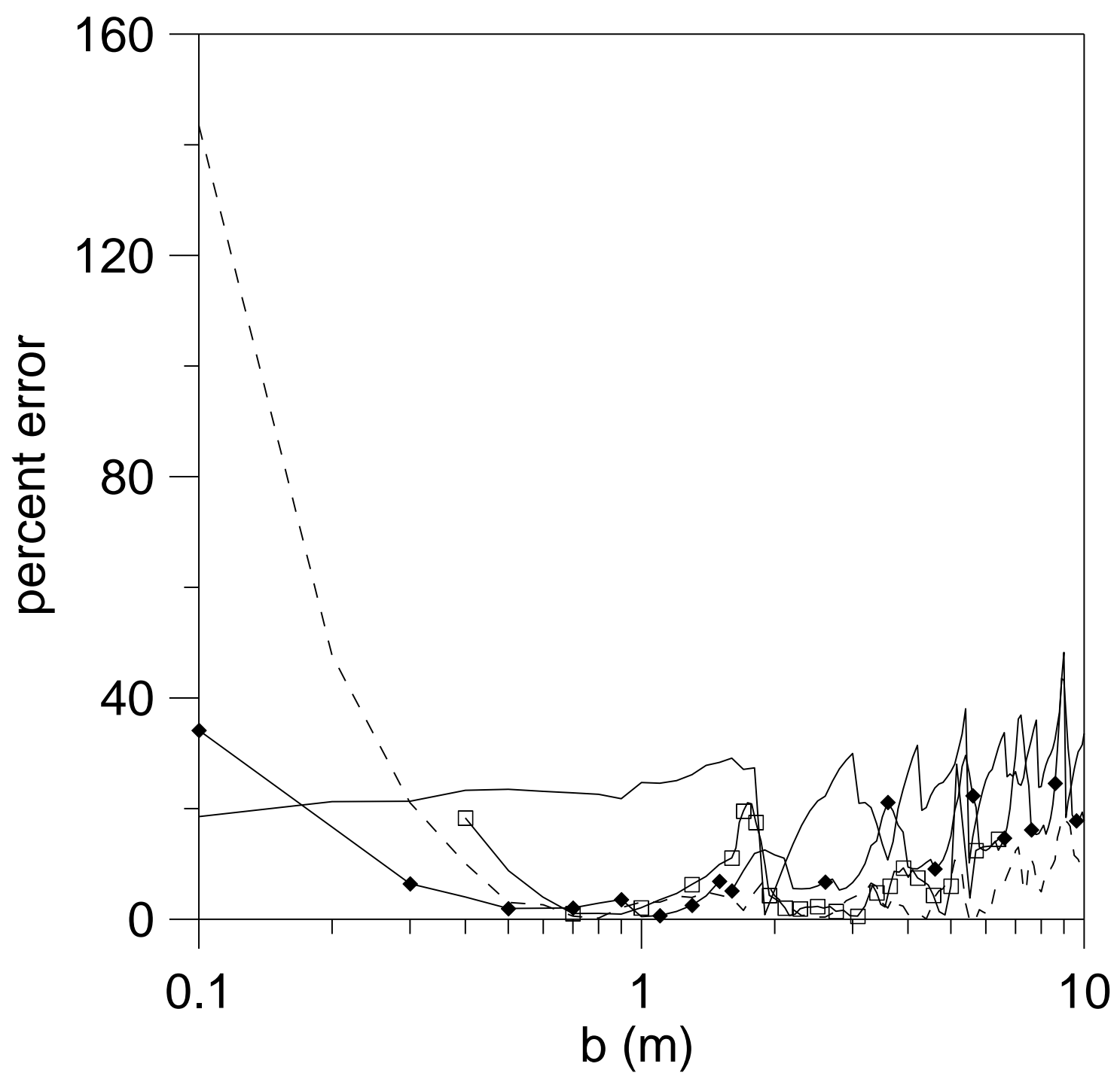

Fig. 4. Percent error of calculated reflection coefficient against the FEM solution for a planar slope with $\mathrm{f}=0.5 \mathrm{~Hz} ; \longrightarrow-=$ present equations, $\longrightarrow \longrightarrow$ Madsen and Sørensen (1992), - - - = Nwogu (1993), — —— = Madsen et al. (2006). 
(a)

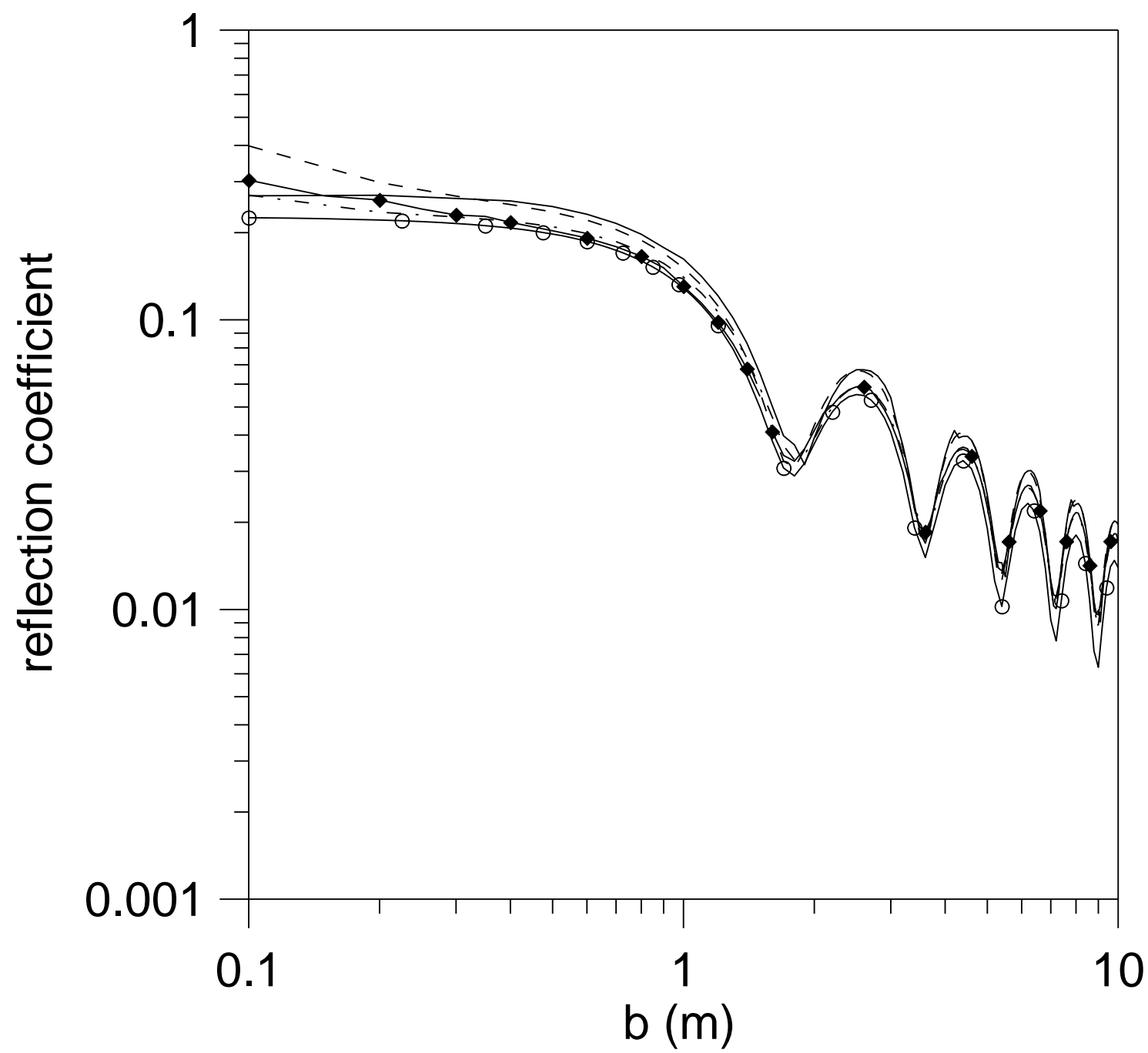

Fig. 5. Variation of reflection coefficients with width of a planar slope with the inclusion of higher-order bottom effects: (a) $\mathrm{f}=0.5 \mathrm{~Hz}$, (b) $\mathrm{f}=0.75 \mathrm{~Hz}$, (c) $\mathrm{f}=1.0 \mathrm{~Hz} ;-\mathrm{O}-=$ Laplace equation, $\longrightarrow-=$ present equations, $\longrightarrow=$ Madsen and Sørensen (1992), - - - - Madsen and Sørensen $(1992)+|\nabla h|^{2}, \cdots-\cdot-=$ Madsen and Sørensen $(1992)+\nabla^{2} h$. 
(b)

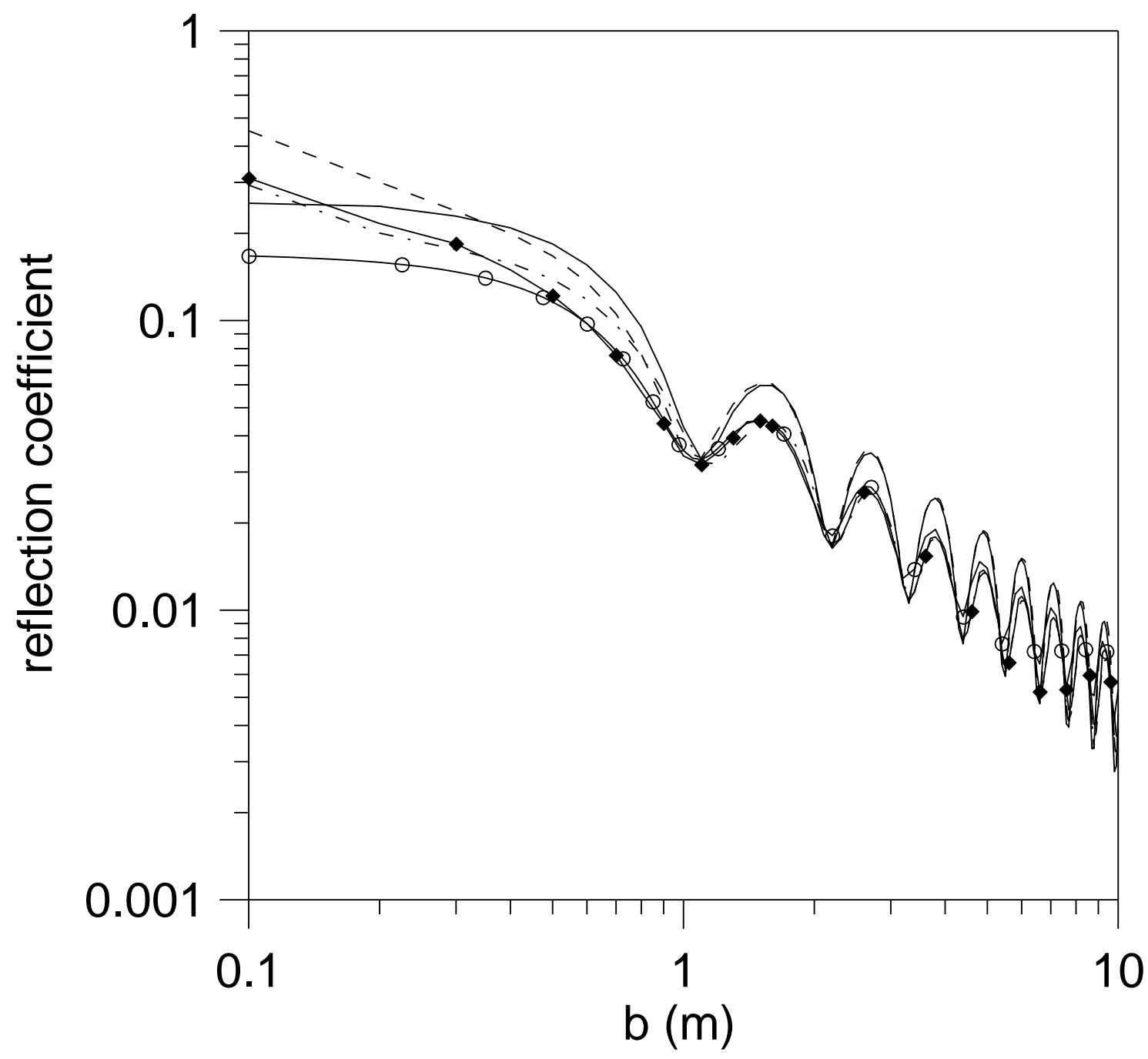

Fig. 5 (continued). 
(c)

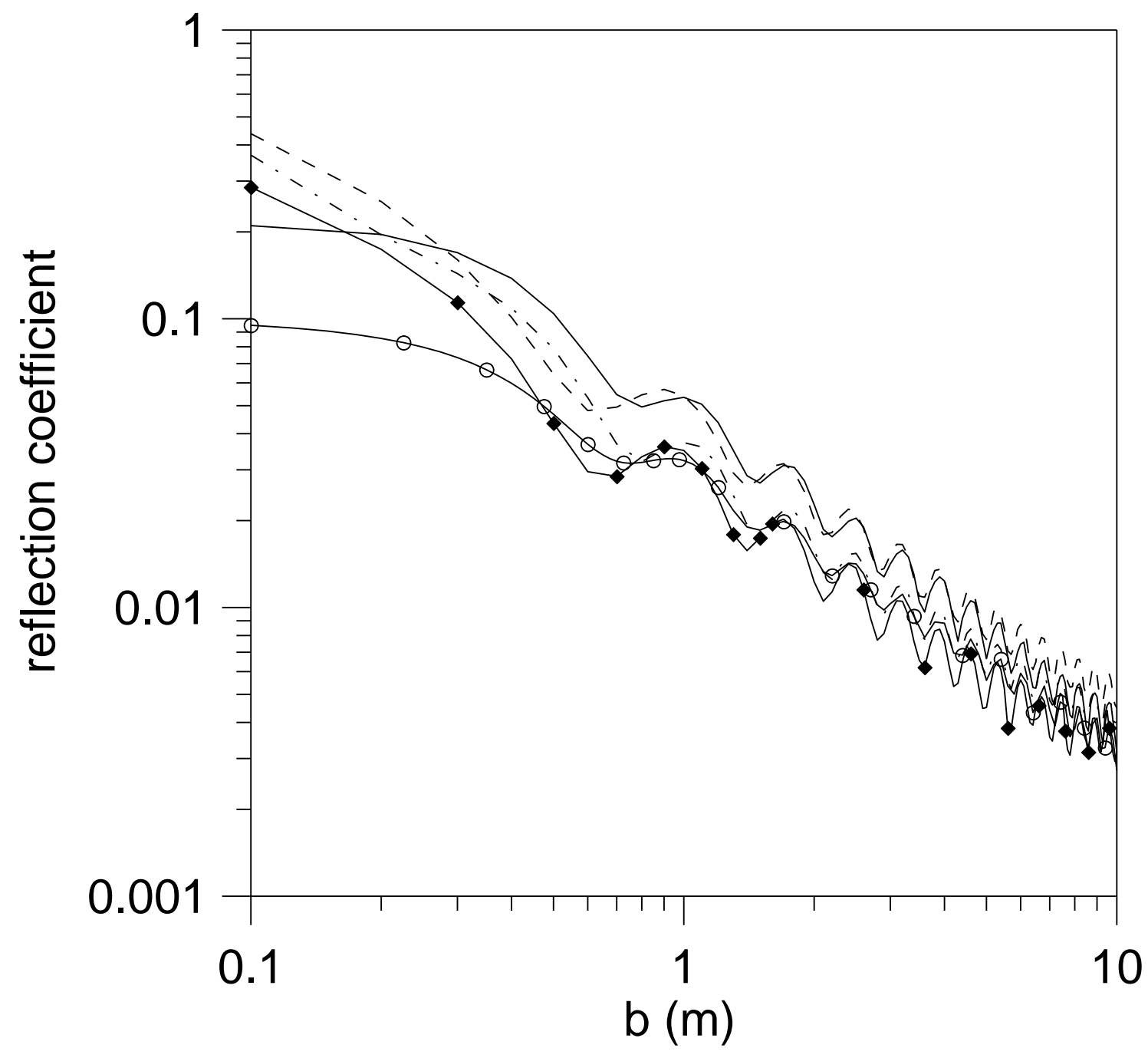

Fig. 5 (continued). 


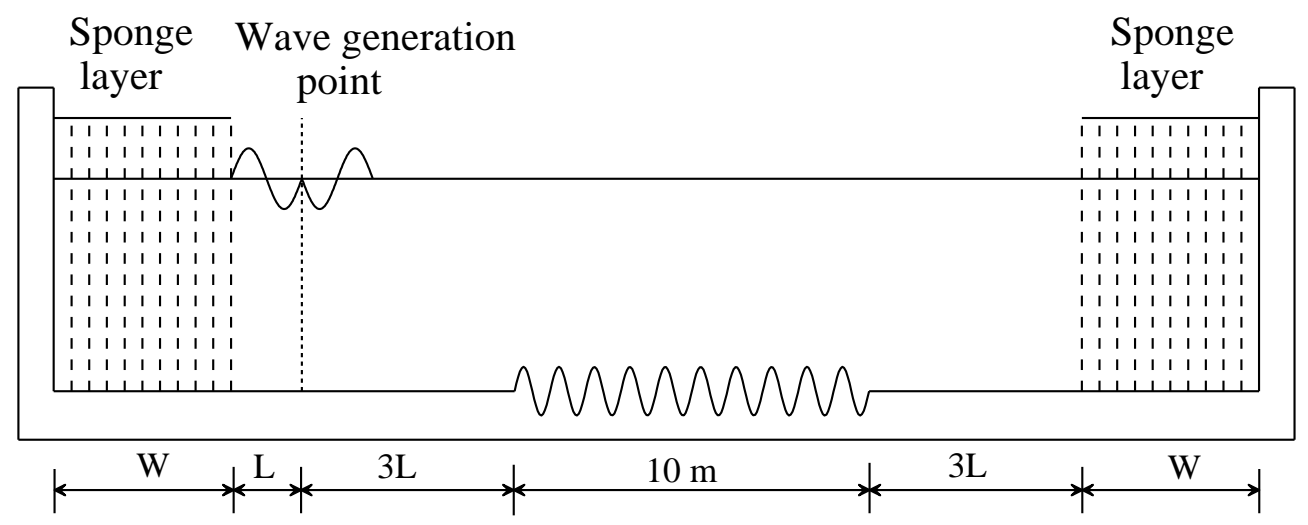

Fig. 6. Computational domain for numerical test of waves propagating over a ripple patch $(n=10)$. 
(a)

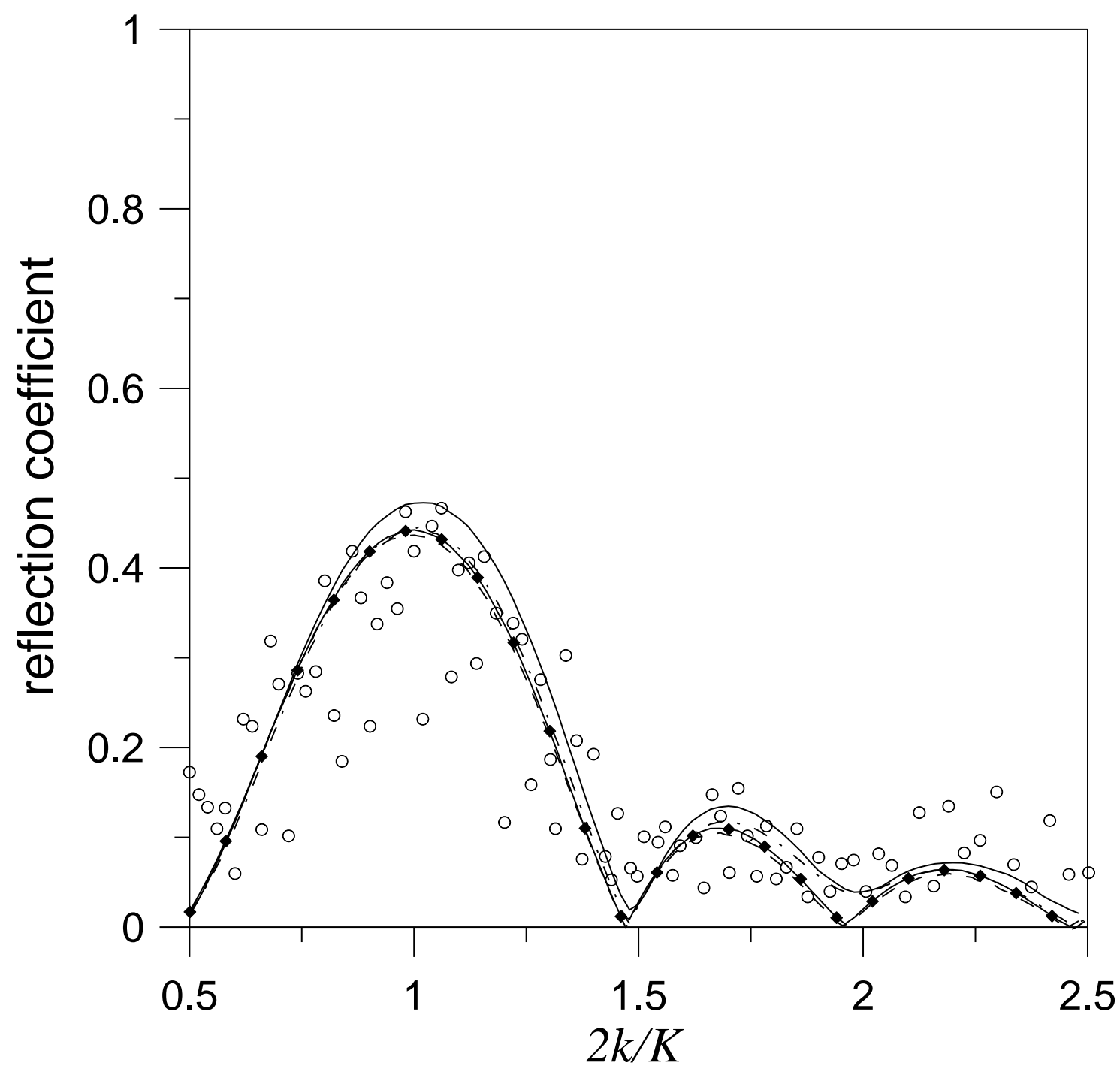

Fig. 7. Variation of reflection coefficient with $2 k / K$ for a ripple bed: (a) $n=2$, (b) $n=4$, (c) $n=10$; $\circ$ $=$ Davies and Heathershaw's (1984) experimental data, $--=$ present equations, $-\longrightarrow=$ Madsen and Sørensen (1992), - - - - Nwogu (1993), — - - = Madsen et al. (2006) (only in Fig. $\underline{7(\mathrm{c})) .}$ 
(b)

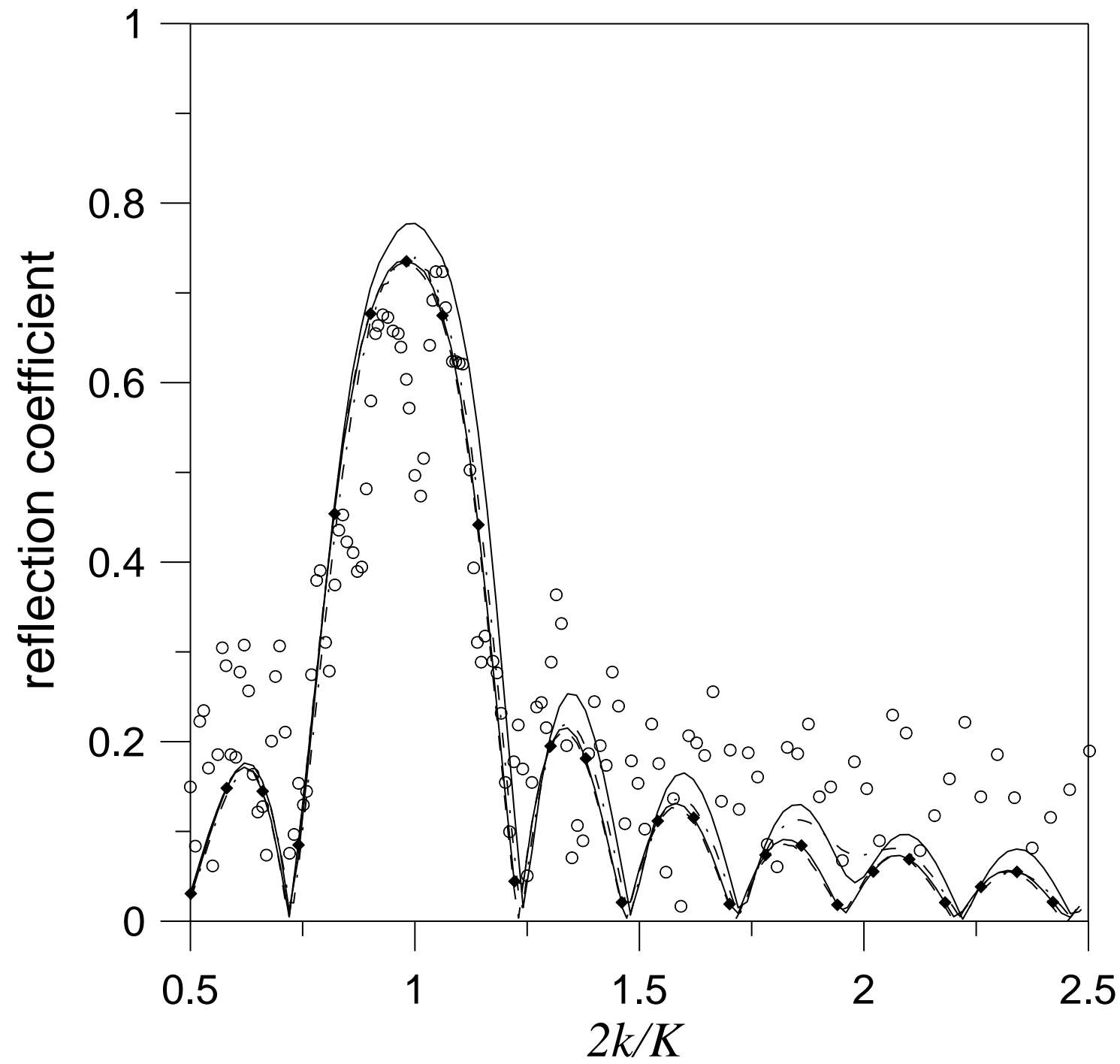

Fig. 7 (continued). 
(c)

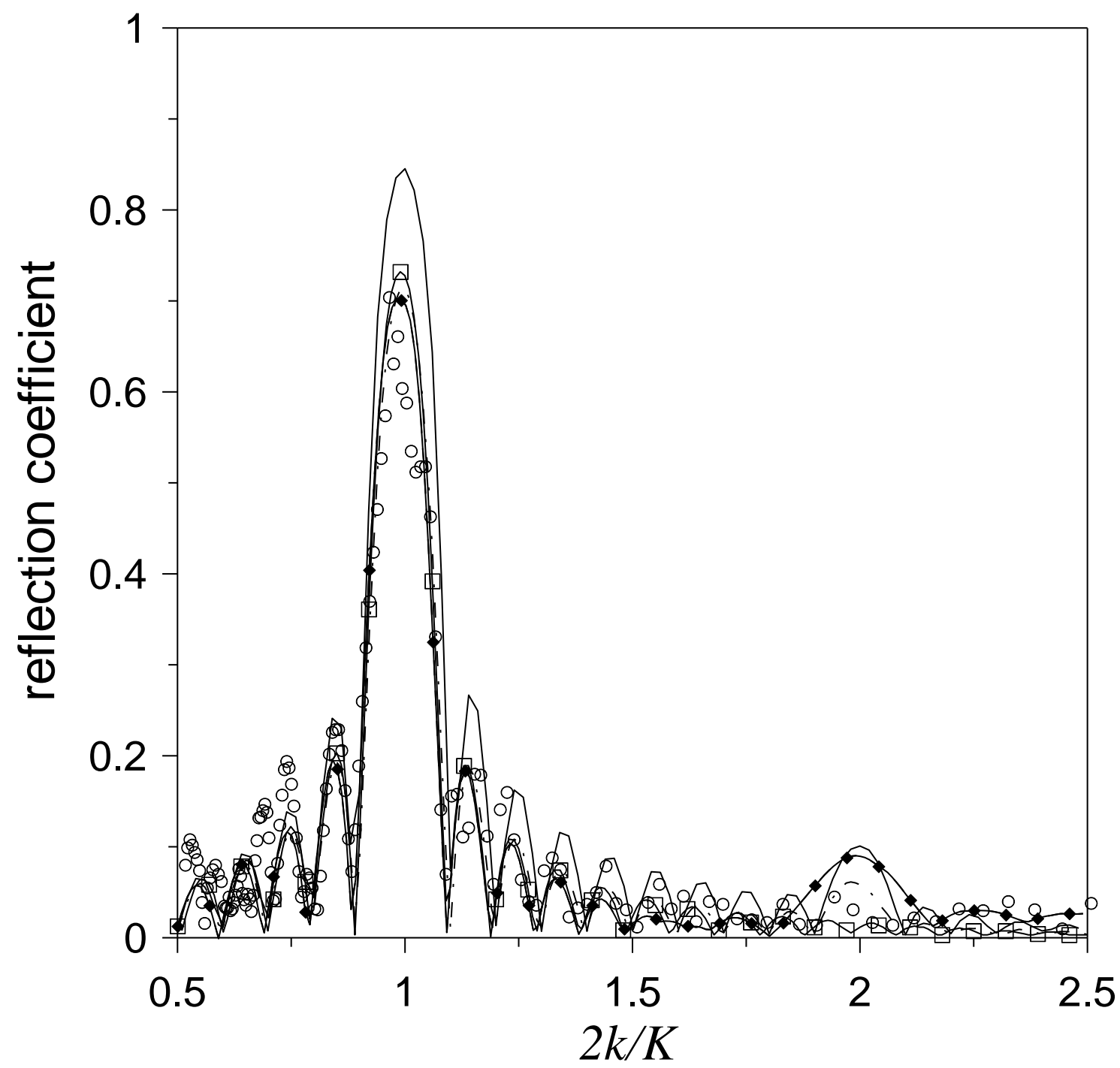

Fig. 7 (continued). 


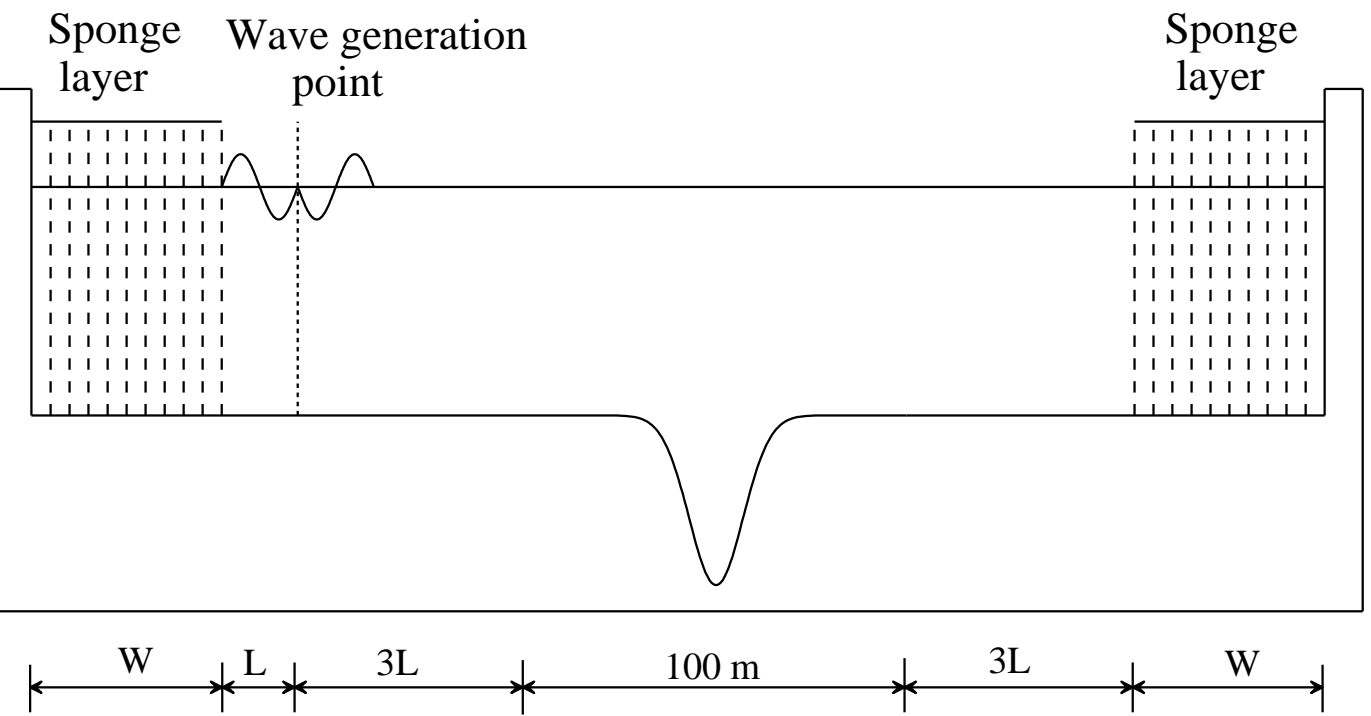

Fig. 8. Computational domain for numerical test of waves propagating over a Gaussian shaped trench. 


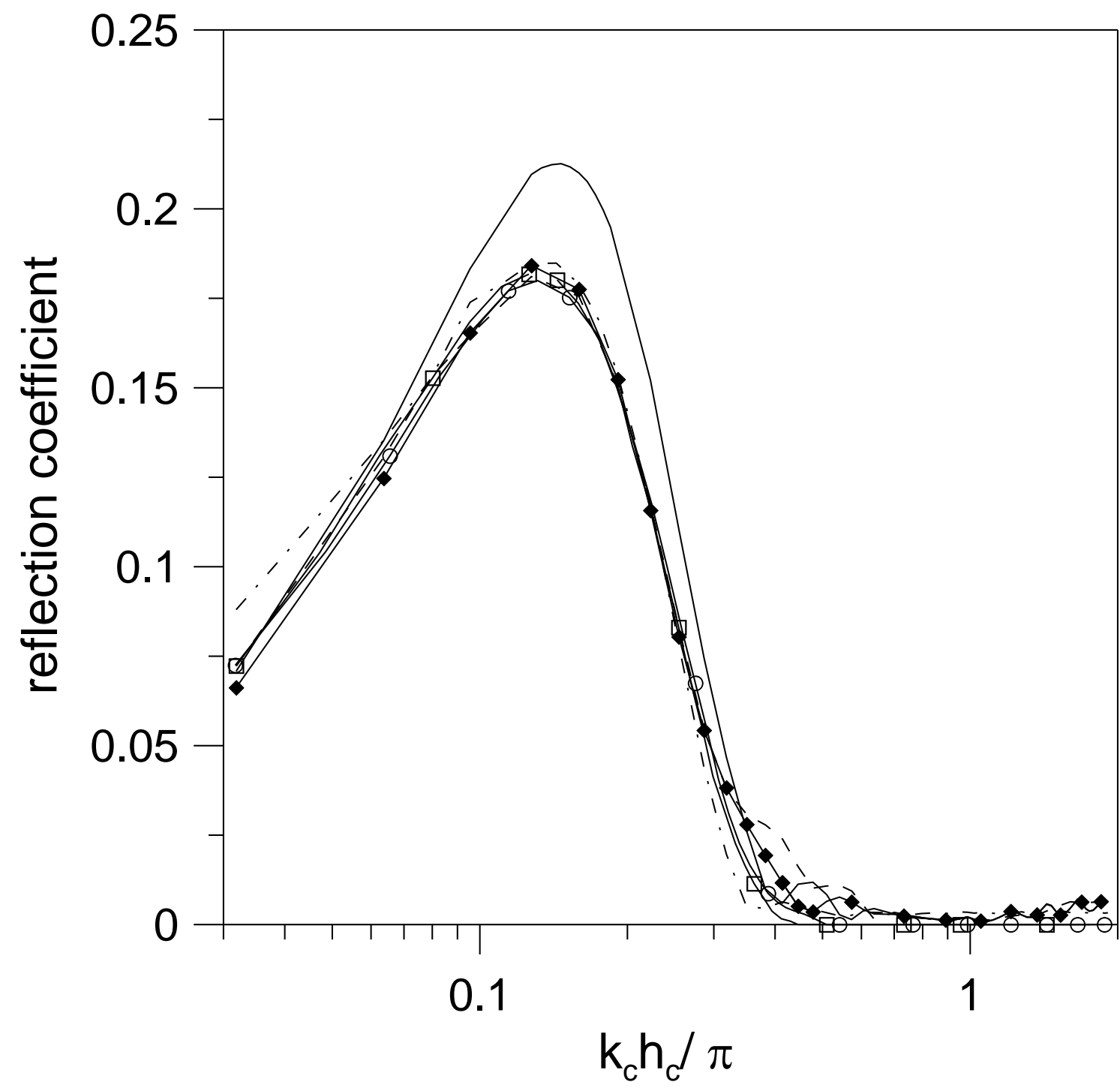

Fig. 9. Variation of reflection coefficients with relative water depth for a Gaussian shaped trench; $-\circ-=$ Bender and Dean (2003), $-\longrightarrow=$ present equations, $-\longrightarrow=$ Madsen and Sørensen (1992), - - - = Nwogu (1993), — - - = Madsen et al. (2006). 